\title{
Environmental Factors and the Risk of Brain Tumours in Young People: A Systematic Review
}

\author{
Angela Zumel-Marne ${ }^{\mathrm{a}-\mathrm{c}}$ Gemma Castano-Vinyals ${ }^{\mathrm{a}-\mathrm{d}}$ Michael Kundi ${ }^{\mathrm{e}}$ \\ Juan Alguacil ${ }^{c, f}$ Elisabeth Cardis ${ }^{a-c}$ \\ ${ }^{a}$ Radiation Programme, ISGlobal, Barcelona, Spain; ${ }^{b}$ Universitat Pompeu Fabra, Barcelona, Spain; ${ }^{c} \mathrm{CIBER}$ \\ Epidemiología y Salud Pública, Madrid, Spain; ${ }^{d}$ IMIM (Hospital del Mar Medical Research Institute), Barcelona, Spain; \\ e Center for Public Health, Department of Environmental Health, Medical University Vienna, Vienna, Austria; ${ }^{\mathrm{f} C e n t r o}$ \\ de Investigación en Recursos Naturales, Salud y Medio Ambiente, Universidad de Huelva, Huelva, Spain
}

\section{Keywords}

Brain tumours · Children · Risk factors · Brain cancer ·

Young adults

\begin{abstract}
Background: Brain tumours (BT) are one of the most frequent tumour types in young people, although little is known about their risk factors. Objective: The objective of the current work was to review and summarize the scientific literature concerning exposure to environmental factors and BT risk in young people ( $<25$ years old). Methods: PUBMED, Embase, Cochrane Library, Scopus, IME-Biomedina (bibliographic database of Consejo Superior de Investigaciones Científicas) and Web of science databases were searched. A score to assess the quality of the methodological information was created. Results: Some possible associations between BT risk in young people were reported for cadmium, consumption of well water, presence of nitrate or nitratenitrogen in tap water, mother's passive smoking, air pollution, parental handling of pesticides at home and/or profes-
\end{abstract}

() 2019 S. Karger AG, Basel sional pesticide treatment within houses, living on a farm and/or with farm animals, some parental occupations and high amount of meat consumption. Conclusions: Although many of the studies reviewed suggest associations between the environmental exposures and BT in children and young adults, at present no reliable conclusion can be drawn as most results are based on small number of cases and exposure assessment is limited. Large-scale studies with better exposure assessment are needed to shed light on these possible associations, especially on exposure to heavy metals, tab water consumption, pesticides and parental smoking.

(c) 2019 S. Karger AG, Basel

\section{Introduction}

Brain tumours (BT) are the second most common cancer type in children and young adults. Around 215,000 cancers in children (0-14 years old) are diagnosed in the world every year, and about 85,000 among adolescents and young adults (15-19 years old), according to esti-
KARGER

E-Mail karger@karger.com

www.karger.com/ned
Angela Zumel-Marne 
mates from the International Agency for Research on Cancer [1]. The incidence rates vary by country from 1.15 to 5.14 cases per 100,000 persons in paediatric populations [2]. The incidence also varies by ethnicity [3].

Little is known about risk factors for BT, with genetic factors and ionising radiation being currently the only well-established risk factors [4]. Migrant studies showed no difference in BT incidence between the offspring of migrants and those of the local population [5], suggesting that environmental exposures might play an important role in BT aetiology [6]. Associations with specific environmental factors, such as heavy metals; pesticides; contaminants in tap/well water or air pollution, have been suggested to play a role in the aetiology of the disease in young people [7-12]. Additionally, other factors affecting children like mother's diet during pregnancy, environmental tobacco smoke or parental occupation have been suggested as potential risk factors for BT in young people, but results are inconsistent $[7,8,13]$.

The main objective of this paper was to review and summarize the scientific literature about exposure to environmental factors and BT risk in young people.

\section{Materials and Methods}

Several searches were made by 1 reviewer (A.Z.-M.) in the following databases: PUBMED, Embase, Cochrane Library, Scopus, Google Scholar, Web of Science and IME (bibliographic database of Consejo Superior de Investigaciones Científicas). Additional articles were found searching the bibliographic references section of articles found (handpicking). Search terms were grouped using the Boolean operators "OR" and "AND." The advanced searches were population AND exposure AND outcome (detailed searches are at the online suppl. Material; for all online suppl. material, see www. karger.com/doi/10.1159/000500601). The keywords used for population were child* OR adolescen* OR young adult OR infan* OR pediatric OR paediatric. The keywords searched for exposure were heavy metal OR cadmium (Cd) OR lead OR tap water OR nitrate OR nitrite OR disinfection byproduct OR meat OR diet OR pesticide OR farm OR parental occupation* OR air pollution OR toxic* OR tobacco smoke OR smoking cigarette OR pregnancy OR cell phone OR mobile (because the literature on tumour risk from ionising radiation exposures is regularly reviewed by various national and international committees we did not include this exposure in our review). The keywords used regarding outcomes were brain cancer OR brain tumo* OR brain neoplasm OR meningioma OR glioma OR primitive neuroectodermal tumo* OR medulloblastoma OR primitive neuroectodermal tumour (PNET) OR central nervous system tumo*. The asterisk $\left(^{*}\right)$ is a wildcard used by the search engines to identify all occurrences that start with the indicated term and are followed by any other letter or letters. For Google scholar we did different searches using a combination of keywords because we could not do the complete search directly (online suppl. material). No restriction for publication year or lan-

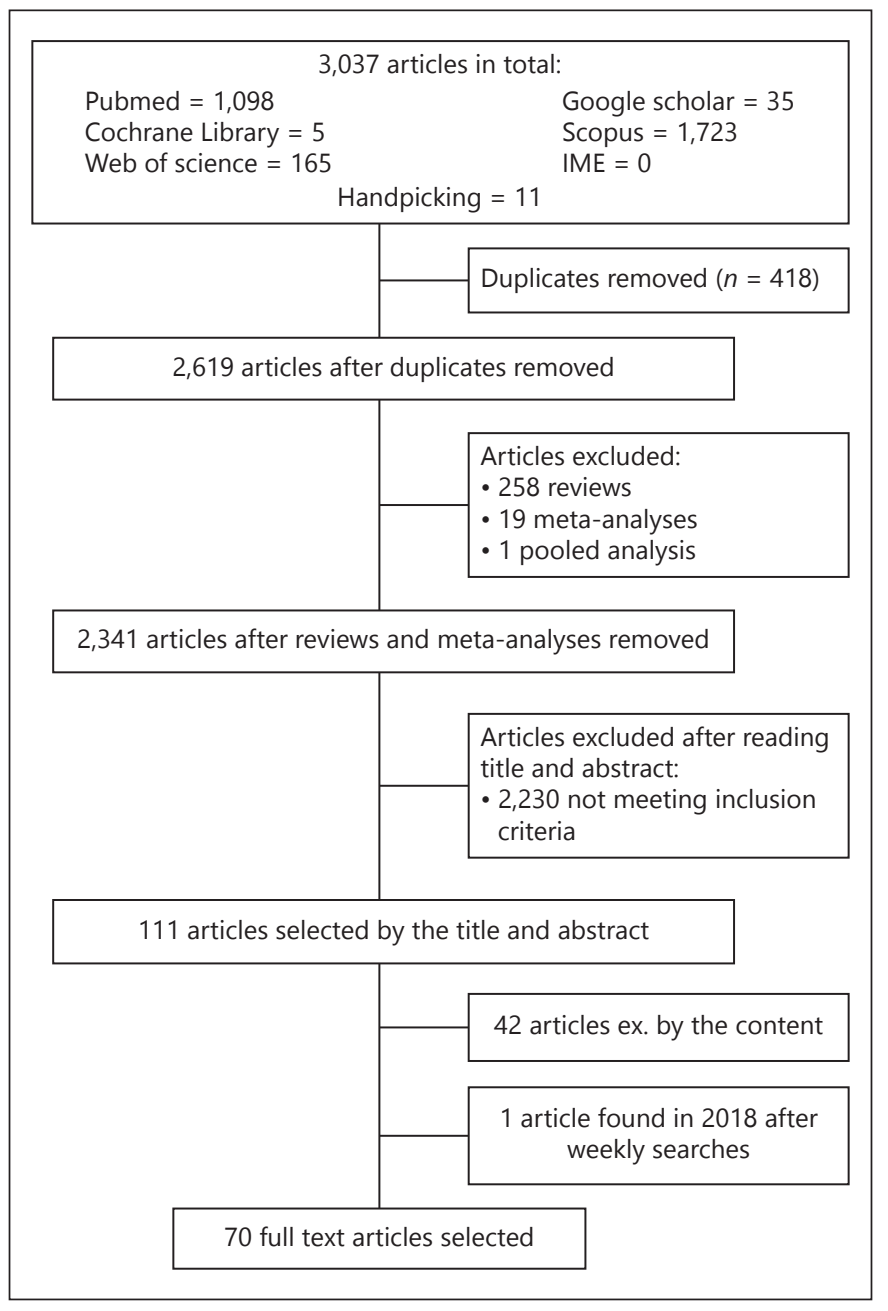

Fig. 1. Flowchart of article selection.

guage was applied. The first searches were performed in July 2016 with additional weekly searches in PUBMED until January 2019.

All articles found were entered into a database using Zotero reference manager $(n=3,037$; Fig. 1$)$. To select the articles related to our topics of interest, we first deleted duplicates $(n=418)$. Then, we excluded the reviews $(n=258)$, meta-analyses $(n=19)$ articles and pooled analysis $(n=1)$, although we checked them whether we had missed any articles. Two thousand three hundred and fortyone titles and abstracts were read to remove articles not meeting our inclusion criteria. We used the following inclusion criteria:

- Studies including young people ( $<25$ years old),

- Studies assessing pre- or post-natal exposure to environmental risk factors,

- Original articles about BT in general or for some specific BT types. Articles that included central nervous system tumours in general, and provided no results for BT, were excluded.

With the removal of 2,230 articles, we read 111 whole papers. Forty-two articles were further excluded because they did not meet our inclusion criteria. One article was added in 2018 after periodical searches, leaving 70 articles for the final analysis. Co-au- 
thors independently assessed the studies for inclusion. Discrepancies were resolved by a final discussion of the research group.

To classify the completeness of the methodological information reported in each article, we created a score (online suppl. Table 3) using the 9 main items of methods of the Strengthening the Reporting of Observational Studies in Epidemiology statement [14]. Each sub-item scored as “+” if it was included or "-" if not included. Additionally, the PRISMA checklist was completed (online suppl. Table 4). No review protocol has been published.

We subdivided the results section into 8 parts: heavy metals, tap water consumption, parental cigarette smoking, air pollution (mainly related to vehicle traffic), exposures related to farms (such as pesticide exposure and contact with animals), non-ionising radiation (such as mobile phones and power lines), parental occupation, and diet (especially meat consumption). Each article was analysed by exposure period: preconception, pregnancy or childhood. When feasible, we also analysed exposure of mother, father or postpartum of the child separately. We also stratified by specific type of pollutant, chemical, or specific occupation, or other exposure categories, as available.

For those exposures that were considered sufficiently homogenous across studies, that is, using similar exposure categories with exposure assessment by comparable methods, we performed DerSimonian-Laird random effect meta-analyses. The random effects model was chosen because the data sources, the age ranges and study designs varied. The $I^{2}$ statistic was applied to report heterogeneity from Cochran's Q test. $I^{2}$ reflects the proportion of total variance estimated to be attributable to between-study heterogeneity. Studies with $I^{2}$ values of $<25,25-50,50-75$ and $>75 \%$ were defined as no, low, moderate and high heterogeneity, respectively. Forest plots of BT risk estimate for mother's smoking (online suppl. Fig. 1), mother's passive smoking (online suppl. Fig. 2), pesticides (online suppl. Fig. 3), living on a farm (online suppl. Fig. 4) and meat consumption (online suppl. Fig. 5) were performed. Effect estimates are relative risks (OR, HR or incident rate ratio) with 95\% CIs. Heterogeneity $I^{2}$ and $p$ value are shown in all figures. Tumor type and exposure period are shown in the right columns.

\section{Results}

From the 70 articles included in this review, 69\% $(n=$ $49)$ had $>200$ cases recruited. Nevertheless, only $23 \%(n=$ 16) of the articles exceeded 500 cases. Most studies (50\%) were conducted in the United States and Canada, the rest were from different countries all over the world, in particular Australia, and European countries. Table 1 provides descriptive information for each study included, classified by type of epidemiological design, with information on the number of participants, age range and geographic and/or ethnic origin of the population.

Table 2 summarizes the main results of the articles grouped by risk factor, indicating the exposure assessment method, the exposure period and the number of cases and controls included in the analyses.

Environmental Factors and the Risk of BT in Young People: A Systematic Review

\section{Heavy Metals}

Only one case - control study [11] was available on the association between heavy metals and BT in young people. Based on only 4 BT cases, Sherief et al. [11] reported significantly higher levels of $\mathrm{Cd}$ in cases than controls $(p<$ 0.001 ) in all biological samples tested: blood, urine, scalp hair and nails.

\section{Tap Water Consumption}

Three case - control studies of the association between water consumption and BT in children and young adults ( $<20$ years old) were found. The first found no increased risk of $\mathrm{BT}$ among children whose mothers reported drinking well water (a hole drilled into the earth) exclusively during their pregnancies. Analyses by region, however, showed an increased risk in the Seattle region (OR 2.6 [1.35.2]) and a decreased risk in Los Angeles County (OR 0.2 [0.1-0.8]). An inverse association with borderline statistical significance was found with the presence of $>10 \mathrm{mg} / \mathrm{L}$ of nitrate in the tap water, while an increased BT risk, based only on 13 cases and 3 controls exposed, was found for nitrite levels above $1 \mathrm{mg} / \mathrm{L}$ (OR 8.8 [2.1-46]) [15].

A further study by the same group [10], including some of the same regions as in Mueller et al. [15] and others from Europe and Canada, confirmed the findings in the US regions and found an increased risk in Canada. No association was found between BT and nitrates or nitrite concentrations overall though an increased $O R$ was found for nitrite concentration $>5 \mathrm{mg} / \mathrm{L}$ after excluding bottled water users. Analysis by tumour type showed an increased risk for nitrite (with no indication of a dose - response) only for astroglial tumours.

The third case - control study [16] found an increased risk of malignant BTs, and central nervous system tumour was found for having lived in a municipality with exposures $>0.31 \mathrm{mg} / \mathrm{L}$ of nitrate-nitrogen $(>1.37 \mathrm{mg} / \mathrm{L} \mathrm{ni-}$ trate) in drinking water compared to lower exposures. The study examined the possible interaction with the presence of calcium and magnesium in the water with high levels of nitrate-nitrogen, assuming that the main source of calcium and magnesium exposure was local drinking water, but no significant association was found.

\section{Parental Cigarette Smoking}

Twenty-two case - control [7, 17-36] and 2 cohort studies $[37,38]$ were found examining the association between cigarette smoking. Among the 20 articles that explored maternal active smoking during preconception or pregnancy and BT risk, 2 case-control studies found no association with childhood BT. Ten case - control 
Table 1. Descriptive information about each article included

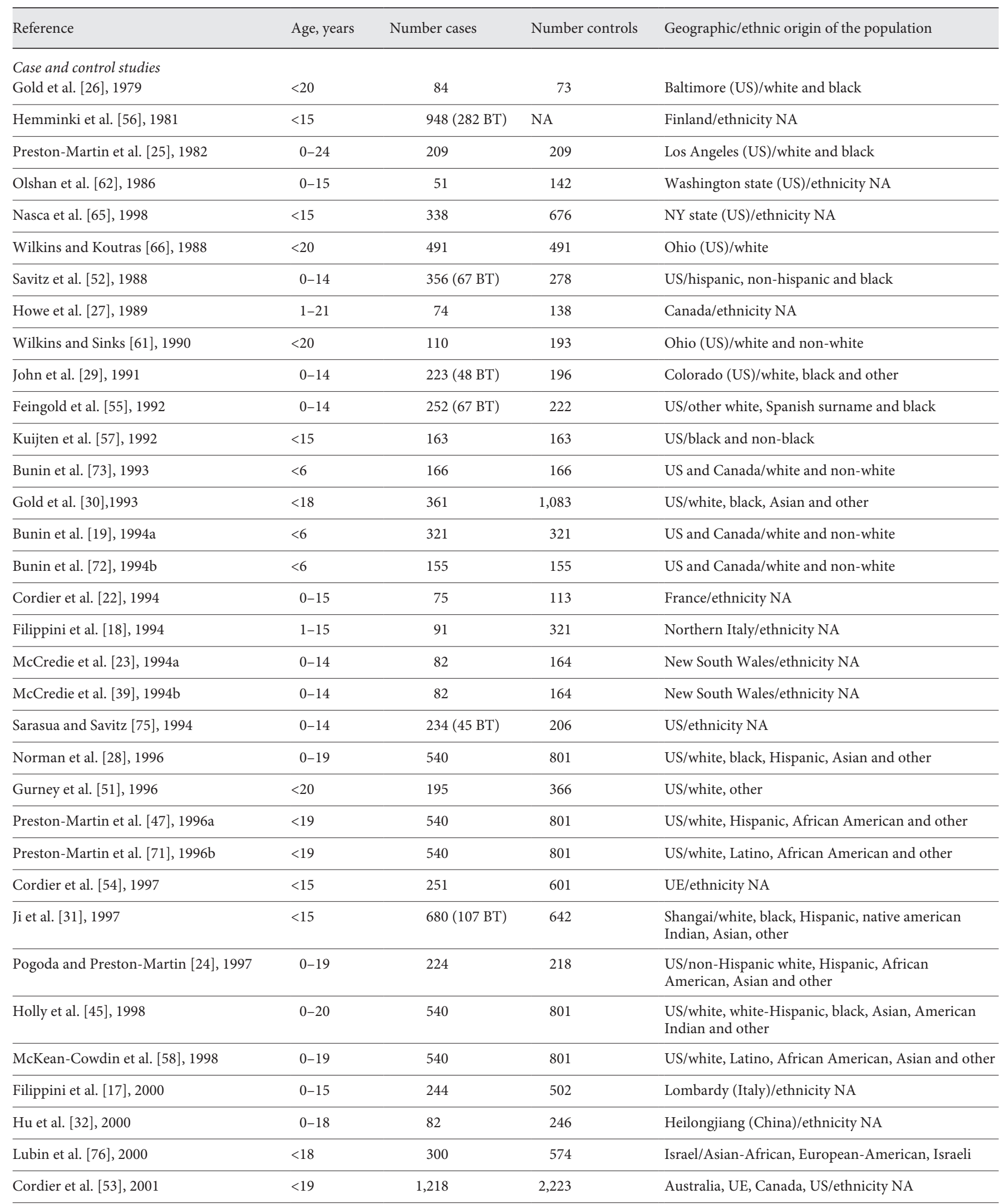


Table 1. (continued)

\begin{tabular}{|c|c|c|c|c|}
\hline Reference & Age, years & Number cases & Number controls & Geographic/ethnic origin of the population \\
\hline Mueller et al. [15], 2001 & $<20$ & 540 & 801 & $\begin{array}{l}\text { US, Europe, Canada and Australia/white, black, } \\
\text { Hispanic, Asian, native American and other }\end{array}$ \\
\hline Pogoda and Preston-Martin [70], 2001 & $<19$ & 540 & 801 & US/white, black, Hispanic, Asian and other \\
\hline Schüz et al. [42], 2001 & $<15$ & 466 & 2,458 & Germany/ethnicity NA \\
\hline Efird et al. [44], 2003 & $0-19$ & 1,213 & 2,213 & US, Europe, Canada and Australia/ethnicity NA \\
\hline Van Wijngaarden et al. [63], 2003 & $0-6$ & 321 & 321 & US and Canada/white and non-white \\
\hline Cordier et al. [7], 2004 & $0-19$ & 1,218 & 2,223 & Australia, Canada, US and Europe/ethnicity NA \\
\hline Mueller et al. [10], 2004 & $<20$ & 836 & 1,485 & US, Europe, Australia/ethnicity NA \\
\hline Bunin et al. [77], 2006 & $<6$ & 315 & 315 & US and Canada/white, black, Hispanic and other \\
\hline Plichart et al. [34], 2008 & $<15$ & 209 & 1,681 & France/ethnicity NA \\
\hline Rosso et al. [41], 2008 & $<6$ & 318 & 318 & US/non-Hispanic white, non-Hispanic black, other \\
\hline Pogoda et al. [69], 2009 & $0-19$ & 1,218 & 2,223 & $\begin{array}{l}\text { Europe, US, Canada, Australia/Latino, other white, } \\
\text { black, and other }\end{array}$ \\
\hline Shim et al. [43], 2009 & $0-10$ & 263 & 263 & Atlantic Coast/white, African American and other \\
\hline Saito et al. [50], 2010 & $0-14$ & 55 & 99 & Japan/Asian \\
\hline Aydin et al. [48], 2011 & $7-19$ & 352 & 646 & $\begin{array}{l}\text { Denmark, Norway, Sweden and Switzerland/ethnicity } \\
\text { NA }\end{array}$ \\
\hline Barrington-Trimis et al. [35], 2013 & $<10$ & 202 & 286 & $\begin{array}{l}\text { US/African-American, non-Hispanic white, Hispa- } \\
\text { nic, Asian and other }\end{array}$ \\
\hline Greenop et al. [9], 2013 & $0-14$ & 374 & 1,467 & Australia/European and non-European \\
\hline Keegan et al. [64], 2013 & $<15$ & 11,119 & 11,039 & Britain/ethnicity NA \\
\hline Peters et al. [60], 2013 & $0-14$ & 306 & 950 & Australia/European and non-European \\
\hline Greenop et al. [78], 2014 & $<15$ & 293 & 726 & Australia/European and non-European \\
\hline Peters et al. [59], 2014 & $0-14$ & 306 & 950 & Australia/European and non-European \\
\hline Sherief et al. [11], 2015 & $3-14$ & 350 (4 BT) & 350 & Egypt/ethnicity NA \\
\hline Von Ehrenstein et al. [12], 2015 & $<6$ & 183 & 30,569 & $\begin{array}{l}\text { California/non-Hispanic white, Hispanic of any } \\
\text { race and other }\end{array}$ \\
\hline Danysh et al. [40], 2016 & $0-5$ & 315 CNS & 1,575 & Texas/non-Hispanic white, Hispanic and other \\
\hline Vienneau et al. [36], 2016 & $7-19$ & 352 & 646 & US/ethnicity NA \\
\hline
\end{tabular}


Table 1. (continued)

\begin{tabular}{|c|c|c|c|}
\hline Reference & Age, years & Number controls & Geographic/ethnic origin of the population \\
\hline \multicolumn{4}{|l|}{ Cohort studies } \\
\hline Kristensen et al. [67], 1996 & $0-14$ & $323,359(182 \mathrm{BT})$ & Norway/ethnicity NA \\
\hline Brooks et al. [38], 2004 & $<15$ & $1,441,942(480 \mathrm{BT})$ & Sweden/ Nordic and other countries classification \\
\hline Tettamanti et al. [37], 2016 & $<23$ & $2,577,305(1,039 \mathrm{BT})$ & Sweden/Nordic and other \\
\hline Spycher et al. [68], 2017 & $<16$ & $2,129,264(227$ CNST) & Switzerland/ethnicity NA \\
\hline \multicolumn{4}{|l|}{ Ecological study } \\
\hline Danysh et al. [8], 2015 & $<15$ & 1,949 & $\begin{array}{l}\text { Texas/non-Hispanic white, non-Hispanic black, } \\
\text { Hispanic and other }\end{array}$ \\
\hline
\end{tabular}

BT, brain tumours; CNST, central nervous system tumours; NA, no available.

studies and 2 cohorts studies found indications of increased risk in the offspring, though the increase was only statistically significant in 2 of the case - control studies $[17,18]$ and in 1 of the cohorts studies (HR 1.24 [1.1-1.53], based on 144 exposed cases) [38]. Additionally, 6 studies found non-statistically significant reduced risks. The overall meta-analytical estimate of the effect of mothers' active smoking is a relative risk of 1.09 (1.00$1.18)$, with little heterogeneity $\left(I^{2}=16 \%\right.$; online suppl. Fig. 1).

Regarding mothers' passive smoking (including exposed to fathers' smoking) before conception or during pregnancy, 14 studies suggested an increased risk in their offspring, being statistically significant in 7. One article studied the association of the exposure in conjunction with paternal occupational exposure to polycyclic aromatic hydrocarbons with a significant increased risk of BT [7]. Two other studies found non-statistically significant reduced risks of BT $[19,20]$. There was a significantly increased meta-analytical effect estimate of 1.32 (1.121.55) for mothers' passive smoking but with moderate heterogeneity $\left(I^{2}=56 \%\right.$; online suppl. Fig. 2$)$.

When examining direct exposure of the child, 2 articles $[21,39]$ reported no association between early infancy or childhood passive smoking and BT (one based on a large sample size). Another study [22] found a significantly increased risk of BT for passive smoking exposure during childhood (OR 2.3 [1.1-4.6]; based on 41 cases).

\section{Air Pollution}

Two case - control studies were retrieved evaluating the link between air pollution and BT. In the first [12], a significantly increased risk of specific types of BT was found in relation to some pollutants, including acetaldehyde, butadiene or polycyclic aromatic hydrocarbons measured in outdoor air of the residence of the child during pregnancy or the first year of life. The second [40] found an increased risk of ependymoma and PNET in offspring of mothers who resided at time of birth within $500 \mathrm{~m}$ of a major roadway and a significantly increased risk of ependymoma for offspring of mothers who resided in areas with high traffic density at time of birth [40]. In an ecological study [8], researchers found a significantly increased risk of astrocytomas in areas with concentrations of 1,3-butadiene between 0.031 and $0.046 \mu \mathrm{g} / \mathrm{m}^{3}$, and of BT in general in areas with diesel particulate matter between 0.218 and $0.784 \mu \mathrm{g} / \mathrm{m}^{3}$ compared to areas with lower concentrations $(<0.218 \mu \mathrm{g} /$ $\mathrm{m}^{3}$ ). No increased risk was observed, however, in areas with highest diesel particulate matter concentrations (> $\left.1.426 \mu \mathrm{g} / \mathrm{m}^{3}\right)$.

\section{Pesticides Exposure and Living on a Farm}

We found 11 articles studying the relationship between BT in young people and pesticide exposure, and 8 focusing more generally on living on a farm as a surrogate for the many exposures that may be encountered in such settings.

Seven studies $[9,19,22-25,41]$ that assessed exposure to pesticides during pregnancy suggested an increased risk of BT, in particular astrocytoma and high-grade glioma, but not PNET (when subtypes were considered). The increases were statistically significant in 4 of the studies $[9,23,24,41]$, and the highest OR was seen for exposure to pesticides when mothers were professionals (OR 4.5 [1.39-15.14] for high-grade gliomas, in a small group of 6 cases and 116 controls) [9]. The overall meta- 
Table 2. Summary of results by exposure

\begin{tabular}{|c|c|c|c|c|c|c|}
\hline Exposure & Reference & Exposure ass. mezthod & Exposure period & Risk factor & $\begin{array}{l}\text { Cases/controls } \\
\text { included in the } \\
\text { analysis }\end{array}$ & Results \\
\hline \multicolumn{7}{|c|}{ Case and control studies } \\
\hline \multirow[t]{5}{*}{$\begin{array}{l}\text { Heavy } \\
\text { metals }\end{array}$} & $\begin{array}{l}\text { Sherief et al. } \\
{[11], 2015}\end{array}$ & Serum, urine, hair, nails & Childhood & $\mathrm{Cd}$ & $4 / 350$ & $\begin{array}{l}\text { Cd concentration in cases is }>\text { than } \\
\text { controls }(p<0.001)\end{array}$ \\
\hline & \multirow{4}{*}{$\begin{array}{l}\text { Mueller et al. } \\
{[15], 2001}\end{array}$} & \multirow[t]{2}{*}{ Face-to-face interview } & \multirow[t]{2}{*}{ Pregnancy } & \multirow[t]{2}{*}{ Well water only } & $18 / 16$ & OR $2.6(1.3-5.2)$ in Seattle \\
\hline & & & & & $2 / 11$ & OR $0.2(0.1-0.8)$ in Los Angeles area \\
\hline & & Water samples of & Before pregnancy & Any nitrate measured & $15 / 41$ & OR $0.6(0.3-1.1)$ \\
\hline & & residences & & Any nitrite measured & $13 / 3$ & OR $8.8(2.1-46)$ \\
\hline \multirow[t]{9}{*}{ Water } & \multirow[t]{7}{*}{$\begin{array}{l}\text { Mueller et al. } \\
{[10], 2004}\end{array}$} & \multirow[t]{2}{*}{ Face-to-face interview } & \multirow{2}{*}{$\begin{array}{l}\text { Pregnancy and } \\
\text { child's first } \\
\text { year of life }\end{array}$} & \multirow[t]{2}{*}{ Well water only } & $45 / 83$ and $134 / 281$ & $\begin{array}{l}\text { OR } 6.1(1.4-27.0) \text { in Canada and } \\
\text { OR } 3.0(1.4-6.2) \text { in Seattle }\end{array}$ \\
\hline & & & & & $304 / 315$ & OR $0.1(0.1-0.9)$ in Los Angeles area \\
\hline & & \multirow[t]{5}{*}{ Water samples } & \multirow{5}{*}{$\begin{array}{l}\text { Pregnancy and } \\
\text { child's first } \\
\text { year of life }\end{array}$} & $>50 \mathrm{mg} / \mathrm{L}$ of nitrate & $14 / 28$ & $\begin{array}{l}\text { OR } 1.5(0.6-3.8) \text { bottled water } \\
\text { users excluded }\end{array}$ \\
\hline & & & & $>5 \mathrm{mg} / \mathrm{L}$ of nitrite & $6 / 8$ & $\begin{array}{l}\text { OR } 5.2(1.2-23.3) \text { bottled water } \\
\text { users excluded }\end{array}$ \\
\hline & & & & $1-<5 \mathrm{mg} / \mathrm{L}$ of nitrite & $151 / 537$ & OR 4.3 (1.4-12.6) only for astroglial tumours \\
\hline & & & & $>5+\mathrm{mg} / \mathrm{L}$ of nitrite & $151 / 537$ & OR 5.7 (1.2-27.2) only for astroglial tumours \\
\hline & & & & $>9.3 \mathrm{mg} / \mathrm{L}$ of $\mathrm{Mg}$ in drinking water & $152 / 132$ & OR $1.24(0.79-1.95)$ if $>0.31 \mathrm{mg} / \mathrm{L}$ of nitrate \\
\hline & \multirow{2}{*}{$\begin{array}{l}\text { Weng et al. } \\
{[16], 2011}\end{array}$} & \multirow{2}{*}{$\begin{array}{l}\text { Water samples of tap } \\
\text { water in residences }\end{array}$} & \multirow{2}{*}{$\begin{array}{l}\text { Childhood and } \\
\text { adolescence }\end{array}$} & $>0.31 \mathrm{mg} / \mathrm{L}$ of nitrate-nitrogen & $190 / 229$ & OR $1.40(1.07-1.84)$ for $\mathrm{MBT}, \mathrm{CN}$ \\
\hline & & & & $>34.8 \mathrm{mg} / \mathrm{L}$ of $\mathrm{Ca}$ in drinking water & $152 / 132$ & OR $1.30(0.84-1.99)$ if $>0.31 \mathrm{mg} / \mathrm{L}$ of nitrate \\
\hline \multirow[t]{23}{*}{$\begin{array}{l}\text { Cigarette } \\
\text { smoking }\end{array}$} & $\begin{array}{l}\text { Gold et al. } \\
{[26], 1979^{*}}\end{array}$ & Interview (NA type) & Before pregnancy & Maternal smoking & $\begin{array}{l}5(+/-) / 1(-/+) \\
\text { discordant pairs }\end{array}$ & OR $5(p=0.22)$ \\
\hline & $\begin{array}{l}\text { Preston-Martin } \\
\text { et al. [25], 1982* }\end{array}$ & Telephone interview & Pregnancy & Maternal passive smoking & $\begin{array}{l}58(+/-) / 39(-/+) \\
\text { discordant pairs }\end{array}$ & OR $1.5(p=0.03)$ \\
\hline & \multirow{2}{*}{$\begin{array}{l}\text { Howe et al. } \\
{[27], 1989}\end{array}$} & \multirow[t]{2}{*}{ Face-to-face interview } & \multirow[t]{2}{*}{ Pregnancy } & Maternal smoking & $18 / 32$ & OR $1.4(0.7-3.0)$ \\
\hline & & & & Paternal smoking & $31 / 58$ & OR $1.13(0.62-2.09)$ \\
\hline & \multirow[t]{2}{*}{$\begin{array}{l}\text { John et al. } \\
{[29], 1991}\end{array}$} & \multirow[t]{2}{*}{ Face-to-face interview } & Year prior to birth & Maternal passive smoking & NA & $\begin{array}{l}\text { OR } 1.6(0.7-3.5) \text { from environmental } \\
\text { father smoking tobacco }\end{array}$ \\
\hline & & & $\begin{array}{l}\text { First trimester of } \\
\text { pregnancy }\end{array}$ & Maternal smoking & NA & OR $0.7(0.3-1.7)$ \\
\hline & \multirow{2}{*}{$\begin{array}{l}\text { Gold et al. } \\
{[30], 1993}\end{array}$} & \multirow[t]{2}{*}{ Face-to-face interview } & Pregnancy & Paternal smoking $>1$ year & $65 / 185$ & OR $1.1(0.7-1.8)$ for astrocytic tumours \\
\hline & & & $\begin{array}{l}\text { During year index } \\
\text { was born }\end{array}$ & Maternal smoking $>1$ year & $64 / 217$ & OR $1.0(0.7-1.4)$ for astrocytic tumours \\
\hline & \multirow[t]{3}{*}{$\begin{array}{l}\text { Bunin et al. } \\
{[19], 1994 a^{*}}\end{array}$} & \multirow[t]{3}{*}{ Thelephone interview } & Pregnancy & Maternal smoking & $60-64 / 58-63$ & $\begin{array}{l}\text { OR } 1.0(0.6-1.7) \text { for astrocytomas } \\
\text { or PNET }\end{array}$ \\
\hline & & & & Mother passive smoking & $83 / 83$ & OR $0.9(0.6-1.5)$ for astrocytomas \\
\hline & & & & & $79 / 81$ & OR $0.9(0.8-1.2)$ for PNET \\
\hline & Cordier et al. & Face-to-face interview & Pregnancy & Maternal smoking & $19 / 23$ & OR $1.6(0.7-3.5)$ \\
\hline & {$[22], 1994^{*}$} & & Childhood & Child passive smoking & $41 / 51$ & OR $2.3(1.1-4.6)$ \\
\hline & Filippini et al. & Face-to-face interview & Pregnancy & Maternal smoking & $18 / 59$ & OR $1.7(0.8-3.8)$ \\
\hline & {$[18], 1994$} & & & Maternal passive smoking & $37 / 92$ & OR $2.2(1.1-4.5 ;>2$ h/day exposed $)$ \\
\hline & & & Conceptional & Maternal passive smoking & $38 / 123$ & OR $1.6(0.8-3.2)$ \\
\hline & & & period & Maternal smoking & $38 / 105$ & OR $1.9(1.0-3.9)$ \\
\hline & $\begin{array}{l}\text { McCredie } \\
\text { et al. [23], } \\
1994 a^{*}\end{array}$ & Face-to-face interview & Pregnancy & Maternal passive smoking & $41 / 49$ & $\begin{array}{l}\text { OR } 2.2(1.2-3.8) \text { from environmental } \\
\text { paternal tobacco smoke }\end{array}$ \\
\hline & $\begin{array}{l}\text { McCredie et al. } \\
{[39], 1994 b^{*}}\end{array}$ & Face-to-face interview & Childhood & Child passive smoking & NA & No association \\
\hline & Norman & Face-to-face interview & Pregnancy & Maternal smoking & $92 / 152$ & OR $0.98(0.72-1.30)$ \\
\hline & et al. [28], & & & Maternal passive smoking & $207 / 262$ & OR $1.2(0.9-1.6)$ \\
\hline & & & & Paternal smoking & $174 / 238$ & OR $1.2(0.9-1.5)$ \\
\hline & $\begin{array}{l}\text { Ji et al. } \\
{[31], 1997}\end{array}$ & Face-to-face interview & Before conception & $\begin{array}{l}\text { Father smoker and mother non } \\
\text { smoker }\end{array}$ & $21 / 17$ & OR 2.7 (0.8-9.9) for $>5$ pack-year \\
\hline
\end{tabular}


Table 2. (continued)

\begin{tabular}{|c|c|c|c|c|c|c|}
\hline Exposure & Reference & Exposure ass. mezthod & Exposure period & Risk factor & $\begin{array}{l}\text { Cases/controls } \\
\text { included in the } \\
\text { analysis }\end{array}$ & Results \\
\hline & $\begin{array}{l}\text { Pogoda and } \\
\text { Preston-Martin } \\
{[24], 1997^{*}}\end{array}$ & Face-to-face interview & Pregnancy & Maternal smoking & $32 / 29$ & OR $1.1(0.6-1.9)$ \\
\hline & \multirow{4}{*}{$\begin{array}{l}\text { Filippini } \\
\text { et al. [17], } \\
2000\end{array}$} & \multirow[t]{4}{*}{ Thelephone interview } & \multirow[t]{2}{*}{ Before pregnancy } & Maternal smoking & $110 / 199$ & OR $1.2(0.9-1.7)$ \\
\hline & & & & Paternal smoking & $155 / 293$ & OR $1.2(0.9-1.7)$ \\
\hline & & & \multirow[t]{2}{*}{ Early pregnancy } & Maternal smoking & $83 / 160$ & OR $1.5(1.0-2.3)$ \\
\hline & & & & $\begin{array}{l}\text { Maternal passive smoking } \\
\text { (never active smoker) }\end{array}$ & $101 / 168$ & OR $1.8(1.2-2.6)$ \\
\hline & \multirow{3}{*}{$\begin{array}{l}\text { Hu et al. [32], } \\
2000\end{array}$} & \multirow[t]{3}{*}{ Face-to-face interview } & Before pregnancy & Maternal passive smoking & $44 / 124$ & OR $1.3(0.8-2.2)$ \\
\hline & & & \multirow[t]{2}{*}{ Pregnancy } & Maternal passive smoking & $46 / 129$ & OR $1.17(0.67-2.04)$ \\
\hline & & & & Maternal smoking & $9 / 18$ & OR $1.20(0.45-3.23)$ \\
\hline & \multirow{4}{*}{$\begin{array}{l}\text { Filippini } \\
\text { et al. [21], } 2002\end{array}$} & \multirow[t]{4}{*}{ Face-to-face interview } & Pregnancy & Maternal smoking & $210 / 422$ & OR $0.9(0.8-1.1)$ \\
\hline & & & \multirow{2}{*}{$\begin{array}{l}\text { Mother regular } \\
\text { exposure }\end{array}$} & \multirow[t]{2}{*}{ Maternal passive smoking } & $706 / 1,257$ & OR $1.1(1.0-1.3)$ any smoking source \\
\hline & & & & & $20 / 162$ & $\begin{array}{l}\text { OR } 1.9 \text { (1.1-3.2) exp. at home for } \\
\text { unspecified glial tumour }\end{array}$ \\
\hline & & & Child 1st year life & Child passive smoking & $564 / 1,087$ & OR $1.0(0.8-1.1)$ \\
\hline & \multirow{6}{*}{$\begin{array}{l}\text { Cordier } \\
\text { et al. [7], } \\
2004\end{array}$} & \multirow{6}{*}{$\begin{array}{l}\text { Face-to-face interview } \\
\text { and job exposure } \\
\text { matrix }\end{array}$} & \multirow[t]{3}{*}{ Pregnancy } & \multirow[t]{3}{*}{ Maternal PAH exposure } & $46 / 90$ & $\begin{array}{l}\text { OR } 1.0(0.7-1.4) \text { PAH exposure from } \\
\text { occupation }\end{array}$ \\
\hline & & & & & $152 / 305$ & $\begin{array}{l}\text { OR } 0.9(0.7-1.2) \text { PAH exposure from } \\
\text { smoking }\end{array}$ \\
\hline & & & & & $13 / 22$ & $\begin{array}{l}\text { OR } 1.2(0.6-2.5) \text { smoking and occupational } \\
\text { exposure }\end{array}$ \\
\hline & & & \multirow[t]{3}{*}{ Preconception } & \multirow[t]{3}{*}{ Paternal PAH exposure } & $484 / 784$ & $\begin{array}{l}\text { OR } 1.3 \text { (1.1-1.6) PAH exposure from } \\
\text { occupation }\end{array}$ \\
\hline & & & & & $273 / 590$ & $\begin{array}{l}\text { OR } 1.1(0.9-1.3) \text { PAH exposure from } \\
\text { smoking }\end{array}$ \\
\hline & & & & & $272 / 646$ & $\begin{array}{l}\text { OR } 1.4(1.1-1.7) \text { smoking and occupational } \\
\text { exposure }\end{array}$ \\
\hline & $\begin{array}{l}\text { Pavlovic } \\
\text { et al. [33], } \\
2005^{*}\end{array}$ & Face-to-face interview & Pregnancy & Maternal smoking & $16 / 24$ & OR $0.6(0.3-1.3)$ \\
\hline & \multirow{4}{*}{$\begin{array}{l}\text { Plichart } \\
\text { et al. [34], } \\
2008\end{array}$} & \multirow[t]{4}{*}{ Telephone interview } & \multirow[t]{2}{*}{ Pregnancy } & \multirow[t]{2}{*}{ Maternal smoking } & $6 / 325$ & OR $1.3(0.5-3.2)$ for astrocytomas \\
\hline & & & & & $10 / 325$ & OR $1.2(0.6-2.4)$ for other gliomas \\
\hline & & & \multirow{2}{*}{$\begin{array}{l}\text { Year prior to } \\
\text { birth }\end{array}$} & \multirow[t]{2}{*}{ Paternal smoking } & $18 / 746$ & OR 3.1 (1.3-7.6) for astrocytomas \\
\hline & & & & & $17 / 746$ & OR $0.8(0.4-1.4)$ for other gliomas \\
\hline & \multirow{4}{*}{$\begin{array}{l}\text { Milne } \\
\text { et al. [20], } \\
2013\end{array}$} & \multirow{4}{*}{$\begin{array}{l}\text { Mailed and thelephone } \\
\text { questionnaires }\end{array}$} & \multirow[t]{2}{*}{ Preconception } & Maternal smoking & $72 / 206$ & OR $0.99(0.70-1.40)$ \\
\hline & & & & Paternal smoking & $74 / 222$ & OR $0.99(0.71-1.32)$ \\
\hline & & & \multirow[t]{2}{*}{ Pregnancy } & Maternal smoking & $51 / 157$ & OR $0.89(0.61-1.31)$ \\
\hline & & & & Paternal smoking & $71 / 202$ & OR $1.04(0.74-1.46)$ \\
\hline & Barrington-Trimis & Face-to-face interview & Pregnancy & Maternal smoking & $21 / 47$ & OR $0.55(0.29-1.05)$ \\
\hline & & & & Paternal smoking & $45 / 57$ & OR $1.03(0.62-1.71)$ \\
\hline & $\begin{array}{l}\text { Vienneau } \\
\text { et al. [36], } \\
2016\end{array}$ & Face-to-face interview & Pregnancy & Maternal smoking & $90 / 133$ & OR $1.23(0.90-1.68)$ \\
\hline Air & Von Ehrenstein & Air toxics info. about & Pregnancy & Acetaldehyde & $34 / 25,361$ & OR 2.30 (1.44-3.67) for PNET \\
\hline pollution & et al. [12], 2015 & participant's residences & & 1,3-Butadiene & $38 / 27,189$ & OR $2.23(1.28-3.88)$ for PNET \\
\hline & & resources board & & $\mathrm{PAH}$ & $27 / 21,368$ & OR 1.44 (1.15-1.80) for medulloblastomas \\
\hline & & & During first & Ortho-dichlorobenzene & $23 / 18,040$ & OR 3.27 (1.17-9.14) for PNET \\
\hline & & & year of life & 1,3-Butadiene & $31 / 25,688$ & OR 3.15 (1.57-6.32) for PNET \\
\hline & & & & Lead & $20 / 16,680$ & OR $1.40(0.97-2.03)$ for astrocytomas \\
\hline
\end{tabular}


Table 2. (continued)

\begin{tabular}{|c|c|c|c|c|c|c|}
\hline Exposure & Reference & Exposure ass. mezthod & Exposure period & Risk factor & $\begin{array}{l}\text { Cases/controls } \\
\text { included in the } \\
\text { analysis }\end{array}$ & Results \\
\hline & $\begin{array}{l}\text { Danysh et al. [40], } \\
2016\end{array}$ & $\begin{array}{l}\text { Birth certificate registry } \\
\text { about participant's }\end{array}$ & At time of birth & $\begin{array}{l}\text { Mother living within } 500 \mathrm{~m} \text { of } \\
\text { a major roadway }\end{array}$ & $23 / 1,173$ & OR $3.08(0.91-10.42)$ for ependymomas \\
\hline & & residences & & $\begin{array}{l}\text { Maternal residential high roadway } \\
\text { density }\end{array}$ & $9 / 1,173$ & OR $3.04(0.38-24.07)$ for PNET \\
\hline & & & & & $15 / 591$ & OR $4.23(1.20-14.88)$ for ependymomas \\
\hline \multirow[t]{30}{*}{ Pesticides } & $\begin{array}{l}\text { Gold et al. [26], } \\
\text { 1979* }\end{array}$ & Interview (NA type) & Prior to diagnosis & Home use of pesticides & $\begin{array}{l}16(+/-) / 7(-/+) \\
\text { discordant pairs }\end{array}$ & OR $2.3(p=0.1)$ \\
\hline & $\begin{array}{l}\text { Preston-Martin } \\
\text { et al. [25], }\end{array}$ & Telephone interview & Pregnancy & $\begin{array}{l}\text { Often or occasional contact with } \\
\text { pesticides }\end{array}$ & $42 / 29$ & OR $1.50(p=0.08)$ \\
\hline & $1982^{*}$ & & & Home treated with pesticides & $10 / 10$ & OR $1.0(p=0.6)$ \\
\hline & & & Childhood & $\begin{array}{l}\text { Often or occasional contact with } \\
\text { pesticides }\end{array}$ & $26 / 24$ & OR $1.10(p=0.44)$ \\
\hline & & & & Home treated with pesticides & $36 / 42$ & OR $0.90(p=0.29)$ \\
\hline & $\begin{array}{l}\text { Howe et al. } \\
{[27], 1989}\end{array}$ & Face-to-face interview & Prior to diagnosis & Pesticides & $19 / 38$ & OR $0.94(0.47-1.90)$ \\
\hline & Bunin et al. & Telephone interview & Pregnancy & Insect sprays or pesticides & $34 / 26$ & OR $1.5(0.8-2.7)$ for astrocytomas \\
\hline & [19], 1994a* & & & & $31 / 39$ & OR 0.7 (0.4-1.4) for PNET \\
\hline & & & & Home treated with pesticides & $24 / 31$ & OR 0.7 (0.4-1.4) for astrocytomas \\
\hline & & & & & $34 / 33$ & OR 1.0 (0.6-1.9) for PNET \\
\hline & Cordier et al. & Face-to-face interview & Pregnancy & House treated with pesticides & $18 / 21$ & OR $1.8(0.8-4.1)$ \\
\hline & {$[22], 1994^{*}$} & & Childhood & House treated with pesticides & $31 / 36$ & OR $2.0(1.0-4.1)$ \\
\hline & $\begin{array}{l}\text { McCredie et al. } \\
{[23], 1994 a^{*}}\end{array}$ & Face-to-face interview & Pregnancy & House treated with pesticides & $20 / 24$ & OR $2.0(1.0-3.9)$ \\
\hline & $\begin{array}{l}\text { Pogoda and } \\
\text { Preston-Martin }\end{array}$ & Face-to-face interview & Pregnancy & Flea/tick pesticides & $76 / 57 ; 29 / 18$ & OR $1.7(1.1-2.6)$ all ages; OR $2.5(1.2-5.5)$ \\
\hline & et al. $[24], 1997^{*}$ & & & & $33 / 17$ & $\begin{array}{l}\text { OR } 2.2(1.1-4.2) \text { pest. prepared/cleaned } \\
\text { by mother }\end{array}$ \\
\hline & & & Childhood & Flea/tick pesticides & $106 / 102$ & OR $1.0(0.7-1.4)$ \\
\hline & $\begin{array}{l}\text { Schüz et al. } \\
{[42], 2001^{*}}\end{array}$ & $\begin{array}{l}\text { Mailed or telephone } \\
\text { interviews }\end{array}$ & Childhood & $\begin{array}{l}\text { Exposed once per year to } \\
\text { household pesticides }\end{array}$ & $8 / 96 ; 5 / 96$ & $\begin{array}{l}\text { OR } 2.45(1.09-5.45) \text { for astrocytomas; OR } \\
1.14(0.44-2.95) \text { for medulloblastomas }\end{array}$ \\
\hline & & & & Garden treated with pesticides & $16 / 290 ; 13 / 290$ & $\begin{array}{l}\text { OR } 0.91(0.48-1.71) \text { for astrocytomas; OR } \\
0.81(0.43-1.53) \text { for medulloblastomas }\end{array}$ \\
\hline & & & & Exposed to wood preservatives & $53 / 746 ; 39 / 746$ & $\begin{array}{l}\text { OR } 1.91(1.22-3.01) \text { for astrocytomas; OR } \\
1.15(0.74-1.77) \text { for medulloblastomas }\end{array}$ \\
\hline & $\begin{array}{l}\text { Rosso et al. } \\
{[41], 2008}\end{array}$ & Telephone interview & Pregnancy & Father lawn care using insecticides & $105 / 67$ & $\begin{array}{l}\text { OR } 1.6(1.0-2.5) \text { for medulloblastomas or } \\
\text { PNET }\end{array}$ \\
\hline & & & $\begin{array}{l}\text { After children } \\
\text { birth }\end{array}$ & Father lawn care using insecticides & $111 / 70$ & $\begin{array}{l}\text { OR } 1.7 \text { (1.1-2.6) for medulloblastomas or } \\
\text { PNET }\end{array}$ \\
\hline & $\begin{array}{l}\text { Shim et al. } \\
{[43], 2009}\end{array}$ & Telephone interview & $\begin{array}{l}\text { During 2-year } \\
\text { before child's }\end{array}$ & Parental herbicides use & $\begin{array}{l}53 / 27 ; 26 / 24 \\
\text { (discordant pairs) }\end{array}$ & $\begin{array}{l}\text { OR } 1.9(1.2-3.0) \text { for astrocytomas; OR } 1.0 \\
(0.6-1.8) \text { for PNET }\end{array}$ \\
\hline & & & $\begin{array}{l}\text { birth and } \\
\text { occurrence BT }\end{array}$ & Father herbicides use & $\begin{array}{l}40 / 20 ; 21 / 18 \\
\text { (discordant pairs) }\end{array}$ & $\begin{array}{l}\text { OR } 2.0(1.2-3.4) \text { for astrocytomas; OR } 1.1 \\
(0.5-2.0) \text { for PNET }\end{array}$ \\
\hline & & & & $\begin{array}{l}\text { Residential herbicide use + paternal } \\
\text { occupational exposure }\end{array}$ & $\begin{array}{l}52(+/-) / 26(-/+) \\
25(+/-) / 21(-/+) \\
\text { (discordant pairs) }\end{array}$ & $\begin{array}{l}\text { OR } 1.9(1.2-3.1) \text { for astrocytomas; OR } 1.2 \\
(0.6-2.1) \text { for PNET }\end{array}$ \\
\hline & & & & $\begin{array}{l}\text { Residential herbicide use by } \\
\text { father + paternal occupational } \\
\text { exposure }\end{array}$ & $\begin{array}{l}41(+/-) / 22(-/+) \\
22(+/-) / 18(-/+) \\
\text { (discordant pairs) }\end{array}$ & $\begin{array}{l}\text { OR } 1.8(1.1-3.1) \text { for astrocytomas; OR } 1.0 \\
(0.5-2.0) \text { for PNET }\end{array}$ \\
\hline & $\begin{array}{l}\text { Greenop et al. } \\
{[9], 2013}\end{array}$ & $\begin{array}{l}\text { Mailed and thelephone } \\
\text { interviews }\end{array}$ & $\begin{array}{l}\text { In year before } \\
\text { pregnancy }\end{array}$ & $\begin{array}{l}\text { Home or garden treated with } \\
\text { pesticides }\end{array}$ & $72 / 187$ & OR $1.54(1.07-2.22)$ \\
\hline & & & Pregnancy & Home or garden treated with & $46 / 116$ & OR $1.52(0.99-2.34)$ \\
\hline & & & & pesticides & $6 / 116$ & OR $4.58(1.39-15.14)$ for high grade gliomas \\
\hline & & & After children & Home or garden treated with & $96 / 303$ & OR $1.04(0.75-1.43)$ \\
\hline & & & birth & pesticides & $39 / 91$ & $\begin{array}{l}\text { OR } 1.63(1.02-2.60) \text { if child was at home } \\
\text { during pest treatment }\end{array}$ \\
\hline
\end{tabular}


Table 2. (continued)

\begin{tabular}{|c|c|c|c|c|c|c|}
\hline Exposure & Reference & Exposure ass. mezthod & Exposure period & Risk factor & $\begin{array}{l}\text { Cases/controls } \\
\text { included in the } \\
\text { analysis }\end{array}$ & Results \\
\hline & \multirow[t]{2}{*}{$\begin{array}{l}\text { Gold et al. } \\
{[26], 1979^{*}}\end{array}$} & \multirow[t]{2}{*}{ Interview (NA type) } & \multirow[t]{2}{*}{ Early childhood } & Living on a farm & $\begin{array}{l}12(+/-) / 3(-/+) \\
\text { discordant pairs }\end{array}$ & OR $4.0(p=0.04)$ \\
\hline & & & & Sick pets & $\begin{array}{l}9(+/-) / 2(-/+) \\
\text { discordant pairs }\end{array}$ & OR $4.50(p=0.07)$ \\
\hline \multirow[t]{25}{*}{$\begin{array}{l}\text { Farm and } \\
\text { animals }\end{array}$} & \multirow[t]{2}{*}{$\begin{array}{l}\text { Bunin et al. } \\
{[19], 1994 a^{*}}\end{array}$} & \multirow[t]{2}{*}{ Telephone interview } & Pregnancy & Living on a farm & $5 / 8 ; 14 / 6$ & $\begin{array}{l}\text { OR } 0.5(0.1-1.8) \text { for astrocytoma; } \\
\text { OR } 3.7(0.8-23.9) \text { for PNET }\end{array}$ \\
\hline & & & $\begin{array}{l}\text { Childhood } \\
\text { (>1 year) }\end{array}$ & Living on a farm & $6 / 9 ; 14 / 6$ & $\begin{array}{l}\text { OR } 0.4(0.1-1.6) \text { for astrocytomas; } \\
\text { OR } 5.0(1.1-46.8) \text { for PNET }\end{array}$ \\
\hline & \multirow{3}{*}{$\begin{array}{l}\text { Cordier } \\
\text { et al. [22], } \\
1994^{*}\end{array}$} & \multirow[t]{3}{*}{ Face-to-face interview } & Pregnancy & Living on a farm & $8 / 2$ & OR $6.7(1.2-38.0)$ \\
\hline & & & & Cat scratches & $10 / 3$ & OR $5.1(1.2-21.6)$ \\
\hline & & & Childhood & Living on a farm & $4 / 2$ & OR $2.5(0.4-16.1)$ \\
\hline & $\begin{array}{l}\text { McCredie } \\
\text { et al. [23], 1994a* }\end{array}$ & Face-to-face interview & Pregnancy & Mother live/work on farm & $5 / 11$ & OR $0.9(0.3-2.6)$ \\
\hline & \multirow{2}{*}{$\begin{array}{l}\text { McCredie } \\
\text { et al. [39], 1994b* }\end{array}$} & \multirow[t]{2}{*}{ Face-to-face interview } & \multirow[t]{2}{*}{ Childhood } & Lived/worked on farm & $4 / 12$ & OR $0.6(0.2-1.9)$ \\
\hline & & & & Contact with horses & $6 / 17$ & OR $0.7(0.3-1.8)$ \\
\hline & \multirow{3}{*}{$\begin{array}{l}\text { Holly } \\
\text { et al. [45], } \\
1998\end{array}$} & \multirow[t]{3}{*}{ Face-to-face interview } & Pregnancy & Lived or worked on a farm & $22 / 21$ & OR $1.6(0.9-2.9)$ \\
\hline & & & & Exposed to pigs & $16 / 11$ & OR $3.8(1.2-12.0)$ \\
\hline & & & Childhood & Lived or worked on a farm & $35 / 36$ & OR $1.5(0.9-2.4)$ \\
\hline & \multirow{5}{*}{$\begin{array}{l}\text { Efird } \\
\text { et al. [44], } \\
2003\end{array}$} & \multirow[t]{5}{*}{ Face-to-face interview } & Pregnancy & Living on a farm & $75 / 119$ & OR $1.3(1.0-1.8)$ \\
\hline & & & & Farm animals & $63 / 95$ & OR $1.4(1.0-1.9)$ \\
\hline & & & Childhood & Living on a farm & $92 / 164$ & OR $1.3(1.0-1.7)$ \\
\hline & & & & Living any farm animal & $82 / 144$ & OR $1.3(0.9-1.8)$ \\
\hline & & & & $\begin{array}{l}\text { Living on a farm starting } \\
<6 \text { months of age }\end{array}$ & $62 / 89$ & OR $1.6(1.1-2.2)$ \\
\hline & \multirow{4}{*}{$\begin{array}{l}\text { Christensen } \\
\text { et al. [46], } \\
2012\end{array}$} & \multirow{4}{*}{$\begin{array}{l}\text { Face-to-face or mailed } \\
\text { interview }\end{array}$} & Pregnancy & Living on a farm & $352 / 38$ & OR $0.40(0.19-0.88)$ \\
\hline & & & Childhood & Living on a farm & $12 / 43$ & OR $0.57(0.28-1.17)$ \\
\hline & & & & Contact with birds & $84 / 206$ & OR $0.7(0.5-0.9)$ \\
\hline & & & $\begin{array}{l}\text { Childhood } \\
\text { (3 years exp.) }\end{array}$ & Goats and sheep & $17 / 57$ & OR $0.5(0.3-0.9)$ \\
\hline & $\begin{array}{l}\text { Savitz } \\
\text { et al. [52], } \\
1988\end{array}$ & $\begin{array}{l}\text { Face-to-face or } \\
\text { thelephone interview }\end{array}$ & Childhood & Electrical and magnetic fields & $25 / 204$ & $\begin{array}{l}\text { OR } 0.82(0.23-2.93) \text { for high } \\
\text { magnetic fields; OR } 0.53(0.15-1.81) \\
\text { for high electric fields }\end{array}$ \\
\hline & \multirow{4}{*}{$\begin{array}{l}\text { Bunin } \\
\text { et al. [19], } \\
1994 a^{*}\end{array}$} & \multirow[t]{4}{*}{ Thelephone interview } & \multirow[t]{4}{*}{ Pregnancy } & Electrically heated waterbed & $32 / 38$ & OR $0.9(0.5-1.7)$ for astrocytomas \\
\hline & & & & & $35 / 34$ & OR $1.0(0.6-1.9)$ for PNET \\
\hline & & & & Electric blanket & $19 / 18$ & OR $1.0(0.5-2.2)$ for astrocytomas \\
\hline & & & & & $21 / 18$ & OR $1.2(0.6-2.5)$ for PNET \\
\hline \multirow{10}{*}{$\begin{array}{l}\text { Non- } \\
\text { ionizing } \\
\text { radiation }\end{array}$} & \multirow{2}{*}{$\begin{array}{l}\text { McCredie } \\
\text { et al. [23], } \\
1994 b^{*}\end{array}$} & \multirow[t]{2}{*}{ Face-to-face interview } & \multirow[t]{2}{*}{ Childhood } & Electric blanket & $6 / 23$ & OR $0.4(0.2-1.2)$ \\
\hline & & & & Electrically heated water bed & $1 / 10$ & OR $0.2(0-1.5)$ \\
\hline & \multirow{4}{*}{$\begin{array}{l}\text { Preston-Martin } \\
\text { et al. [47], } \\
\text { 1996a }\end{array}$} & \multirow[t]{4}{*}{ Face-to-face interview } & \multirow[t]{2}{*}{ Pregnancy } & Electric blanket & $53 / 485$ & OR $0.9(0.6-1.2)$ \\
\hline & & & & Electrically heated waterbeds & $55 / 484$ & OR $0.9(0.6-1.3)$ \\
\hline & & & Childhood & Electric blanket & $28 / 512$ & OR $1.0(0.6-1.7)$ \\
\hline & & & & Electrically heated waterbeds & $22 / 518$ & OR $1.2(0.7-2)$ \\
\hline & $\begin{array}{l}\text { Gurney } \\
\text { et al. [51], } \\
1996\end{array}$ & $\begin{array}{l}\text { Mailed and telephone } \\
\text { interviews }\end{array}$ & Childhood & Magnetic fields & $23 / 52$ & $\begin{array}{l}\text { OR } 0.9(0.5-1.5) \text { for high level of } \\
\text { magnetic fields }\end{array}$ \\
\hline & Schüz & Mailed or telephone & Pregnancy & Electric blanket & $10 / 111$ & OR $1.81(0.85-3.86)$ for astrocytomas \\
\hline & et al. [42], & interviews & & & $1 / 111$ & OR $0.51(0.01-3.83)$ for ependymomas \\
\hline & & & & & $9 / 111$ & OR $2.02(0.95-4.28)$ for medulloblastomas \\
\hline
\end{tabular}


Table 2. (continued)

\begin{tabular}{|c|c|c|c|c|c|c|}
\hline Exposure & Reference & Exposure ass. mezthod & Exposure period & Risk factor & $\begin{array}{l}\text { Cases/controls } \\
\text { included in the } \\
\text { analysis }\end{array}$ & Results \\
\hline & $\begin{array}{l}\text { Saito } \\
\text { et al. [50], } \\
2010\end{array}$ & $\begin{array}{l}\text { Face-to-face interview } \\
\text { and magnetic fields } \\
\text { measurements }\end{array}$ & Childhood & Magnetic fields & $3 / 1$ & $\begin{array}{l}\text { OR } 10.90(1.05-113) \text { for } \geq 0.4 \mu \mathrm{T} \\
\text { in the bedroom }\end{array}$ \\
\hline & Aydin & Face-to-face interview & Childhood & Regular users of mobile phones & $194 / 329$ & OR $1.36(0.92-2.02)$ \\
\hline & $\begin{array}{l}\text { et al. [48], } \\
2011\end{array}$ & & & $\begin{array}{l}\text { Started to use mobile phones } \\
5 \text { years before the diagnosis }\end{array}$ & $46 / 81$ & OR $1.26(0.70-2.28)$ \\
\hline & $\begin{array}{l}\mathrm{Li} \\
\text { et al. [49], } \\
2012\end{array}$ & $\begin{array}{l}\text { From National } \\
\text { Communication } \\
\text { Council }\end{array}$ & $\begin{array}{l}\text { Child exp. } 5 \text { years } \\
\text { prior to diagnosis }\end{array}$ & RF exposure & $114 / 4,923$ & $\begin{array}{l}\text { OR } 1.14(0.83-1.55) \text { for above median } \\
\text { annual power density estimated } \\
\left(168.07 \mathrm{WYs} / \mathrm{km}^{2}\right)\end{array}$ \\
\hline \multirow[t]{33}{*}{$\begin{array}{l}\text { Parental } \\
\text { oc. }\end{array}$} & \multirow{4}{*}{$\begin{array}{l}\text { Hemminki } \\
\text { et al. [56], } \\
1981\end{array}$} & \multirow[t]{4}{*}{$\begin{array}{l}\text { Maternity welfare } \\
\text { district information }\end{array}$} & \multirow[t]{4}{*}{ Pregnancy } & Mother factory worker & 5 discordant pairs & $\begin{array}{l}\text { OR } 8(p<0.05) \text { for cases recruited } \\
1969-1975\end{array}$ \\
\hline & & & & Father machine repair men & 14 discordant pairs & OR $4.39(p<0.05)$ \\
\hline & & & & Father painter & 14 discordant pairs & OR $2.59(p<0.10)$ \\
\hline & & & & & 7 discordant pairs & OR $5.0(p<0.05)$ \\
\hline & \multirow{2}{*}{$\begin{array}{l}\text { Olshan } \\
\text { et al. [62], } \\
1986\end{array}$} & \multirow[t]{2}{*}{ Face-to-face interview } & \multirow{2}{*}{$\begin{array}{l}1 \text { year prior to } \\
\text { birth to time of } \\
\text { diagnosis }\end{array}$} & \multirow[t]{2}{*}{$\begin{array}{l}\text { Father ever employment in the } \\
\text { aerospace industry }\end{array}$} & $8 / 12$ & $\begin{array}{l}\text { OR } 2.10(0.79-5.60) \text { for children } \\
0-9 \text { years old }\end{array}$ \\
\hline & & & & & $1 / 16$ & $\begin{array}{l}\text { OR } 0.12(0.01-1.08) \text { for children } \\
10-15 \text { years old }\end{array}$ \\
\hline & \multirow{2}{*}{$\begin{array}{l}\text { Nasca } \\
\text { et al. [65], } \\
1988\end{array}$} & \multirow[t]{2}{*}{ Thelephone interview } & \multirow[t]{2}{*}{ Postnatal } & \multirow{2}{*}{$\begin{array}{l}\text { Father industrial exposure to } \\
\text { ionizing radiation }\end{array}$} & $8 / 676$ & OR $2.48(1.01-5.57)$ for more exposure \\
\hline & & & & & $21 / 676$ & OR $2.4(1.3-4.3)$ for less exposure \\
\hline & \multirow{8}{*}{$\begin{array}{l}\text { Wilkins and } \\
\text { Koutras }[66] \text {, } \\
1988\end{array}$} & \multirow[t]{8}{*}{$\begin{array}{l}\text { Computerized records } \\
\text { of births and deaths }\end{array}$} & \multirow[t]{8}{*}{ Postnatal } & $\begin{array}{l}\text { Agriculture, forestry and } \\
\text { fishing occupation }\end{array}$ & NA exp. & OR $2.4(1.2-4.9)$ \\
\hline & & & & Father construction occupation & NA exp. & OR $2.3(1.3-4.1)$ \\
\hline & & & & $\begin{array}{l}\text { Father chemicals, drugs and } \\
\text { paints occupation }\end{array}$ & NA exp. & OR $1.9(0.7-5.1)$ \\
\hline & & & & Father metal occupation & NA exp. & OR $1.8(1.1-2.9)$ \\
\hline & & & & Father machinery occupation & NA exp. & OR $1.7(1.1-2.7)$ \\
\hline & & & & Food and tobacco occupation & NA exp. & OR $0.9(0.5-1.8)$ \\
\hline & & & & Medicine and science & $79 / 45$ & OR $0.6(0.2-1.5)$ \\
\hline & & & & $\begin{array}{l}\text { Electrical assembling, installing } \\
\text { and repairing occupations }\end{array}$ & $19 / 9$ & OR $2.7(1.2-6.1)$ \\
\hline & \multirow{3}{*}{$\begin{array}{l}\text { Wilkins and } \\
\text { Sinks [61], } \\
1990\end{array}$} & \multirow[t]{3}{*}{ Thelephone interview } & Pregnancy & Father agriculture occupations & $4 / 491$ & OR $1.6(0.4-6.1)$ \\
\hline & & & Preconception & Father metal employment & $13 / 491$ & OR $3.3(1.3-8.5)$ \\
\hline & & & Preconception & $\begin{array}{l}\text { Father aromatic nitro } \\
\text { compounds exposure }\end{array}$ & NA exp. & OR 5.7 (1.4-23.2) for picric acid exposure \\
\hline & \multirow{6}{*}{$\begin{array}{l}\text { Feingold } \\
\text { et al. [55], } \\
1992\end{array}$} & \multirow{6}{*}{$\begin{array}{l}\text { Job-exposure matrix } \\
\text { and face to } \\
\text { face/telephone } \\
\text { interviews }\end{array}$} & \multirow[t]{6}{*}{ Preconception } & Father exp. to any chemical & $22 / 99$ & OR $0.9(0.4-2.2)$ \\
\hline & & & & Father exp. to hydrocarbons & $18 / 84$ & OR $0.8(0.3-2.0)$ \\
\hline & & & & Father exp. to inorganic compounds & $17 / 54$ & OR $1.2(0.4-3.3)$ \\
\hline & & & & Father exp. to lead & $2 / 12$ & OR $0.7(0.1-3.8)$ \\
\hline & & & & Father exp. to asbestos & $10 / 23$ & OR $1.7(0.6-5.3)$ \\
\hline & & & & Father exp. to physical agents & $14 / 65$ & OR $0.9(0.3-2.3)$ \\
\hline & \multirow{8}{*}{$\begin{array}{l}\text { Kuijten } \\
\text { et al. [57], } \\
1992\end{array}$} & \multirow[t]{8}{*}{ Telephone interview } & \multirow[t]{3}{*}{ Pregnancy } & $\begin{array}{l}\text { Father exp. to transportation } \\
\text { industry }\end{array}$ & $\begin{array}{l}2(+/-) / 14(-/+) \\
\text { discordant pairs }\end{array}$ & OR $0.1(0.0-0.6)$ for astrocytomas \\
\hline & & & & Father exp. to ionizing radiation & $\begin{array}{l}9(+/-) / 8(-/+) \\
\text { discordant pairs }\end{array}$ & OR $1.1(0.4-3.4)$ for astrocytomas \\
\hline & & & & $\begin{array}{l}\text { Father exp. to electromagnetic } \\
\text { fields exp. }\end{array}$ & $\begin{array}{l}8(+/-) / 9(-/+) \\
\text { discordant pairs }\end{array}$ & OR $0.9(0.3-2.6)$ for astrocytomas \\
\hline & & & Preconception & $\begin{array}{l}\text { Father exp. to metal-related } \\
\text { occup. }\end{array}$ & $\begin{array}{l}21(+/-) / 20(-/+) \\
\text { discordant pairs }\end{array}$ & OR $1.1(0.5-2.1)$ for astrocytomas \\
\hline & & & Postnatal & $\begin{array}{l}\text { Father exp. to metal-related } \\
\text { occup. }\end{array}$ & $\begin{array}{l}15(+/-) / 18(-/+) \\
\text { discordant pairs }\end{array}$ & OR $0.8(0.4-1.8)$ for astrocytomas \\
\hline & & & Preconception & $\begin{array}{l}\text { Father exp. to electrical } \\
\text { repairing }\end{array}$ & $\begin{array}{l}8(+/-) / 1(-/+) \\
\text { discordant pairs }\end{array}$ & OR $8.0(1.1-356.1)$ for astrocytomas \\
\hline & & & Postnatal & Father exp. to painting worker & $\begin{array}{l}5(+/-) / 2(-/+) \\
\text { discordant pairs }\end{array}$ & OR $2.5(0.4-26.2)$ for astrocytomas \\
\hline & & & Preconception & Nurse (mother) & $\begin{array}{l}8(+/-) / 1(-/+) \\
\text { discordant pairs }\end{array}$ & $\begin{array}{l}\text { OR } 8.0(1.1-356.1) \text { for cases } 0-4 \text { years } \\
\text { and astrocytomas }\end{array}$ \\
\hline
\end{tabular}


Table 2. (continued)

\begin{tabular}{|c|c|c|c|c|c|c|}
\hline Exposure & Reference & Exposure ass. mezthod & Exposure period & Risk factor & $\begin{array}{l}\text { Cases/controls } \\
\text { included in the } \\
\text { analysis }\end{array}$ & Results \\
\hline & \multirow{4}{*}{$\begin{array}{l}\text { Cordier } \\
\text { et al. [54], } \\
1997\end{array}$} & \multirow[t]{4}{*}{ Face-to-face interview } & \multirow[t]{4}{*}{$\begin{array}{l}\text { Five years before } \\
\text { birth }\end{array}$} & Mother exp. to solvents & \multirow[t]{4}{*}{$251 / 601$} & $\begin{array}{l}\text { OR } 1.3(0.9-2.0) \text { and OR } 2.4(1.2-4.9) \\
\text { for high exposures }\end{array}$ \\
\hline & & & & Mother exp. to $\mathrm{PAH}$ & & OR $0.7(0.4-1.2)$ \\
\hline & & & & Father exp. to solvent & & OR $1.0(0.7-1.5)$ \\
\hline & & & & Father exp. to $\mathrm{PAH}$ & & OR $1.5(1.1-2.1)$ \\
\hline & \multirow[t]{6}{*}{$\begin{array}{l}\text { McKean-Cowdin } \\
\text { et al. [58], } 1998\end{array}$} & \multirow[t]{6}{*}{ Face-to-face interview } & \multirow[t]{6}{*}{$\begin{array}{l}\text { Five years before } \\
\text { birth }\end{array}$} & $\begin{array}{l}\text { Father chemical-petroleum } \\
\text { industry }\end{array}$ & $20 / 24$ & OR 2.1 (1.1-3.9) for astroglial tumours \\
\hline & & & & $\begin{array}{l}\text { Mother chemical-petroleum } \\
\text { industry }\end{array}$ & $13 / 10$ & OR 3.3 (1.4-7.7) for astroglial tumours \\
\hline & & & & Father electrical workers & $34 / 23$ & OR $2.3(1.3-4.0)$ \\
\hline & & & & Father agriculture-farming & $22 / 27$ & OR $1.2(0.7-2.1)$ \\
\hline & & & & Mother agriculture-farming & $5 / 11$ & OR $0.93(0.34-2.60)$ \\
\hline & & & & $\begin{array}{l}\text { Mother material packager and } \\
\text { handler }\end{array}$ & $13 / 14$ & OR $2.3(1.1-5.1)$ \\
\hline & \multirow{13}{*}{$\begin{array}{l}\text { Cordier } \\
\text { et al. [53], } \\
2001\end{array}$} & \multirow[t]{13}{*}{ Face-to-face interview } & \multirow{12}{*}{$\begin{array}{l}\text { During the last } \\
5 \text { years period } \\
\text { before the } \\
\text { child's birth }\end{array}$} & Father agriculture & $80 / 104$ & OR $1.3(1.0-1.8)$ \\
\hline & & & & Mother agriculture occupations & $22 / 39$ & OR $1.1(0.7-1.9)$ \\
\hline & & & & Father petroleum activities & $14 / 10$ & OR 3.4 (1.4-8.2) for astroglial tumours \\
\hline & & & & $\begin{array}{l}\text { Father metal products } \\
\text { occupations }\end{array}$ & $84 / 174$ & OR $0.9(0.7-1.1)$ \\
\hline & & & & $\begin{array}{l}\text { Mother metal products } \\
\text { occupations }\end{array}$ & $7 / 17$ & OR $0.6(0.3-1.6)$ \\
\hline & & & & Father aerospace activities & $30 / 59$ & OR $0.8(0.5-1.3)$ \\
\hline & & & & Mother aerospace activities & $10 / 24$ & OR $0.6(0.3-1.3)$ \\
\hline & & & & Father painting occupations & $31 / 43$ & OR $1.2(0.8-2.0)$ \\
\hline & & & & Mother painting occupations & $9 / 9$ & OR $1.7(0.7-4.5)$ \\
\hline & & & & $\begin{array}{l}\text { Father electrical work } \\
\text { occupations }\end{array}$ & $98 / 168$ & OR $1.1(0.9-1.5)$ \\
\hline & & & & $\begin{array}{l}\text { Mother electrical work } \\
\text { occupations }\end{array}$ & $22 / 36$ & OR $1.3(0.7-2.2)$ \\
\hline & & & & Mother textile activities & $35 / 54$ & OR $1.7(1.1-2.7)$ \\
\hline & & & Preconception & $\begin{array}{l}\text { Mother health services } \\
\text { occupation }\end{array}$ & $57 / 61$ & OR $1.7(1.1-2.4)$ \\
\hline & \multirow{2}{*}{$\begin{array}{l}\text { Van } \\
\text { Wijngaarden } \\
\text { et al. [63], } \\
\text { 2003* }\end{array}$} & \multirow[t]{2}{*}{ Thelephone interview } & \multirow{2}{*}{$\begin{array}{l}\text { During work } \\
\text { history in } \\
\text { general }\end{array}$} & Mother insecticide exposure & $53 / 35 ; 37 / 37$ & $\begin{array}{l}\text { OR } 1.9(1.1-3.3) \text { for astrocytomas; } \\
\text { OR } 1.0(0.6-1.7) \text { for PNET }\end{array}$ \\
\hline & & & & Father farm worker & $21 / 19 ; 28 / 30$ & $\begin{array}{l}\text { OR } 1.1(0.5-2.3) \text { for astrocytomas; } \\
\text { OR } 0.9(0.5-1.7) \text { for PNET }\end{array}$ \\
\hline & \multirow{2}{*}{$\begin{array}{l}\text { Keegan } \\
\text { et al. [64], } \\
2013\end{array}$} & \multirow[t]{2}{*}{$\begin{array}{l}\mathrm{NRCT} \text { and birth } \\
\text { registers }\end{array}$} & \multirow[t]{2}{*}{ Postnatal } & $\begin{array}{l}\text { Father occupational solvents } \\
\text { exposures }\end{array}$ & $44 / 27$ & OR $1.71(0.99-2.93)$ for ependymoma \\
\hline & & & & $\begin{array}{l}\text { Father occupational paints } \\
\text { exposures }\end{array}$ & $74 / 94$ & OR 0.7 (0.5-1.0) for astrocytomas \\
\hline & \multirow{2}{*}{$\begin{array}{l}\text { Peters } \\
\text { et al. [60], } \\
2013\end{array}$} & \multirow[t]{2}{*}{$\begin{array}{l}\text { Mailed and telephone } \\
\text { interviews }\end{array}$} & $\begin{array}{l}\text { Any time } \\
\text { before birth }\end{array}$ & Mother occup. exposure to diesel & $17 / 29$ & OR $2.03(1.09-3.81)$ \\
\hline & & & Preconception & Father occup. exposure to diesel & $53 / 124$ & OR $1.62(1.12-2.34)$ \\
\hline & \multirow{3}{*}{$\begin{array}{l}\text { Peters } \\
\text { et al. [59], } \\
2014\end{array}$} & \multirow[t]{3}{*}{$\begin{array}{l}\text { Mailed and telephone } \\
\text { interviews }\end{array}$} & $\begin{array}{l}\text { Any time } \\
\text { before birth }\end{array}$ & $\begin{array}{l}\text { Mother occup. to chlorinated } \\
\text { solvents }\end{array}$ & $3 / 4$ & OR $8.59(0.79-78.9)$ \\
\hline & & & \multirow[t]{2}{*}{ Preconception } & $\begin{array}{l}\text { Father occup. exposure to } \\
\text { solvents }\end{array}$ & $43 / 92$ & OR $1.55(0.99-2.43)$ \\
\hline & & & & $\begin{array}{l}\text { Father occup. to aromatic } \\
\text { solvents }\end{array}$ & NA exp & OR $1.76(1.10-2.82)$ \\
\hline & $\begin{array}{l}\text { Preston-Martin } \\
\text { et al. [25], } \\
1982^{*}\end{array}$ & Telephone interview & Pregnancy & Meat consumption & $127 / 140$ & $\begin{array}{l}\text { OR } 2.3(p=0.008) \text { for } 2 \text { or more times } \\
\text { per week }\end{array}$ \\
\hline & \multirow{2}{*}{$\begin{array}{l}\text { Bunin } \\
\text { et al. [73], } \\
1993\end{array}$} & \multirow[t]{2}{*}{ Telephone interview } & \multirow[t]{2}{*}{ Pregnancy } & Vegetables & $166 / 166$ & $\begin{array}{l}\text { OR } 0.37(0.19-0.72) \text { for PNET in } \\
\text { higher quartile }\end{array}$ \\
\hline & & & & Bacon & $166 / 166$ & $\begin{array}{l}\text { OR } 1.71 \text { (1.02-2.89) for PNET according } \\
\text { mother's weekly consumption }\end{array}$ \\
\hline
\end{tabular}


Table 2. (continued)

\begin{tabular}{|c|c|c|c|c|c|c|}
\hline Exposure & Reference & Exposure ass. mezthod & Exposure period & Risk factor & $\begin{array}{l}\text { Cases/controls } \\
\text { included in the } \\
\text { analysis }\end{array}$ & Results \\
\hline \multirow[t]{35}{*}{ Diet } & \multirow{4}{*}{$\begin{array}{l}\text { Bunin } \\
\text { et al. [72], } \\
1994 b\end{array}$} & \multirow[t]{4}{*}{ Telephone interview } & \multirow[t]{4}{*}{ Pregnancy } & Cured meats & $41 / 41$ & $\begin{array}{l}\text { OR } 1.7(0.8-3.4) \text { for astrocytic gliomas } \\
\text { in higher quartile }\end{array}$ \\
\hline & & & & Vitamin C & $35 / 35$ & $\begin{array}{l}\text { OR } 0.7(0.4-1.5) \text { for astrocytic gliomas } \\
\text { in higher quartile }\end{array}$ \\
\hline & & & & Iron supplements & NA & OR $0.5(0.3-0.8)$ for astrocytic gliomas \\
\hline & & & & Fruits/juices & $34 / 34$ & $\begin{array}{l}\text { OR } 0.7(0.3-1.4) \text { for astrocytic gliomas } \\
\text { in higher quartile }\end{array}$ \\
\hline & $\begin{array}{l}\text { Cordier } \\
\text { et al. [22], } \\
1994^{*}\end{array}$ & Face-to-face interview & Pregnancy & Cured meat consum. & $63 / 103$ & OR $0.7(0.2-3.0)$ \\
\hline & $\begin{array}{l}\text { McCredie } \\
\text { et al. [23], } \\
1994 a^{*}\end{array}$ & Face-to-face interview & Pregnancy & Cured meat consum. & $29 / 34$ & OR 2.5 (1.1-5.7) for high consumption \\
\hline & \multirow{2}{*}{$\begin{array}{l}\text { Sarasua } \\
\text { et al. [75], } \\
1994\end{array}$} & \multirow[t]{2}{*}{ Face-to-face interview } & Pregnancy & Hot-dogs & $33 / 125$ & OR $2.3(1.0-5.4) 1$ or more times/week \\
\hline & & & Childhood & Hot-dogs & $29 / 93$ & OR $2.1(0.7-6.1) 1$ or more times/week \\
\hline & \multirow{3}{*}{$\begin{array}{l}\text { Preston-Martin } \\
\text { et al. [71], } \\
\text { 1996b }\end{array}$} & \multirow[t]{3}{*}{ Face-to-face interview } & \multirow[t]{3}{*}{ Pregnancy } & \multirow[t]{3}{*}{ Cured meat consum. } & $60 / 60$ & $\begin{array}{l}\text { OR } 2.1(1.3-3.2) \text { for eating at least } \\
\text { twice a day }\end{array}$ \\
\hline & & & & & $47 / 40$ & $\begin{array}{l}\text { OR } 2.2(1.4-3.6) \text { for eating cured meat } \\
\text { and no vitamins were taken }\end{array}$ \\
\hline & & & & & $201 / 377$ & $\begin{array}{l}\text { OR } 0.54(0.39-0.75) \text { for additional } \\
\text { multivitamin consumption during } 3 \\
\text { trimesters }\end{array}$ \\
\hline & \multirow{3}{*}{$\begin{array}{l}\text { Lubin } \\
\text { et al. [76], } \\
2000\end{array}$} & \multirow[t]{3}{*}{ Personal interview } & Pregnancy & Nitrate & $300 / 574$ & OR $1.5(0.8-1.7)$ for high level of intake \\
\hline & & & Childhood & Nitrate & $300 / 574$ & OR $1.2(0.8-1.7)$ for high level of intake \\
\hline & & & Pregnancy & Vitamin C & $300 / 574$ & OR $1.1(0.7-1.7)$ for the highest intake \\
\hline & \multirow{3}{*}{$\begin{array}{l}\text { Pogoda and } \\
\text { Preston-Martin } \\
\text { [70], 2001 }\end{array}$} & \multirow[t]{3}{*}{ Face-to-face interview } & \multirow[t]{3}{*}{ Pregnancy } & \multirow[t]{3}{*}{ Cured meat (nitrite exposure) } & $377 / 601$ & OR $1.2(0.9-1.6)$ for low concentrations \\
\hline & & & & & $27 / 24$ & $\begin{array}{l}\text { OR } 2.3(1.3-4.4) \text { for } 0.50-0.99 \mathrm{mg} \text { of } \\
\text { nitrite average daily }\end{array}$ \\
\hline & & & & & $6 / 10$ & OR $1.3(0.5-3.3)$ for higher concentration \\
\hline & \multirow{4}{*}{$\begin{array}{l}\text { Bunin } \\
\text { et al. [74], } \\
2005\end{array}$} & \multirow[t]{4}{*}{ Telephone interview } & \multirow[t]{4}{*}{ Pregnancy } & Fruits/juices & $n=158$ & OR $0.6(0.3-1.1)$ for PNET/medulloblastoma \\
\hline & & & & Red meat & $n=354$ & $\begin{array}{l}\text { OR } 1.1(0.8-1.7) \text { for more than once } \\
\text { per month }\end{array}$ \\
\hline & & & & French fries & $n=187$ & $\begin{array}{l}\text { OR } 2.4(1.2-4.9) \text { for more than twice } \\
\text { per week }\end{array}$ \\
\hline & & & & Non-fresh peaches or similar & $n=97$ & OR $0.5(0.3-0.8)$ \\
\hline & \multirow{4}{*}{$\begin{array}{l}\text { Bunin } \\
\text { et al. [77], } \\
2006\end{array}$} & \multirow[t]{4}{*}{ Telephone interview } & Periconception & Cured meat and fish & $n=182$ & OR, $1.4(0.8-2.2)$ for $2-3.5$ times/week \\
\hline & & & & & $n=152$ & OR $1.0(0.6-1.9)$ for $>5$ times/week \\
\hline & & & Midpregnancy & Cured meat and fish & $n=176$ & OR $1.1(0.7-1.9)$ for $2-3.5$ times/week \\
\hline & & & Midpregnancy & & $n=154$ & OR $1.0(0.6-1.8)$ for $>5$ times/week \\
\hline & \multirow{9}{*}{$\begin{array}{l}\text { Pogoda } \\
\text { et al. [69], } \\
2009\end{array}$} & \multirow[t]{9}{*}{ Face-to-face interview } & \multirow[t]{9}{*}{ Pregnancy } & Cured meat & $284 / 413 ; 161 / 413$ & OR 1.5 (1.1-2.1) for all BT in higher quartile \\
\hline & & & & & $\begin{array}{l}142 / 2,170 \\
96 / 2,170 \\
199 / 2,170 \\
104 / 2,170\end{array}$ & $\begin{array}{l}\text { OR } 2.5(1.1-5.8) \text { for pilocytic astrocytomas; } \\
\text { OR } 2.1(1.1-4.3) \text { for anaplastic astrocytomas; } \\
\text { OR } 1.8(1.2-2.7) \text { unespecified astrocytomas } \\
\text { OR } 2.0(0.4-2.9) \text { for ependymomas }\end{array}$ \\
\hline & & & & Cruciferous vegetables & $96 / 2,184$ & OR $0.4(0.3-0.7)$ for astrocytomas \\
\hline & & & & Grains & $292 / 526$ & OR $0.9(0.8-0.9)$ for all BT in higher quartile \\
\hline & & & & & $122 / 2,171$ & OR $0.3(0.1-0.6)$ for malignant gliomas \\
\hline & & & & Fresh fish & $237 / 566$ & OR $0.7(0.6-0.9)$ for all BT in higher quartile \\
\hline & & & & Fresh fish & $122 / 2,182$ & OR 0.5 (0.3-0.6) for malignant gliomas \\
\hline & & & & Fresh fish & $96 / 2,182$ & OR $1.6(1.1-2.4)$ for anaplastic astrocytomas \\
\hline & & & & Non-cured meat & $199 / 2,183$ & $\begin{array}{l}\text { OR } 1.2(1.1-1.3) \text { for unspecified } \\
\text { astrocytomas }\end{array}$ \\
\hline & $\begin{array}{l}\text { Greenop } \\
\text { et al. [78], } \\
2014\end{array}$ & Mailed questionnaire & Pregnancy & $\begin{array}{l}\text { Coffee } \\
\text { Tea }\end{array}$ & $\begin{array}{l}150 / 359 \\
189 / 493\end{array}$ & $\begin{array}{l}\text { OR } 1.23(0.92-1.64) \\
\text { OR } 1.0(0.7-1.4)\end{array}$ \\
\hline
\end{tabular}


Table 2. (continued)

\begin{tabular}{|c|c|c|c|c|c|c|}
\hline Exposure & Reference & Exposure ass. method & Exposure period & Risk factor & Exposed cases & Results \\
\hline \multicolumn{7}{|c|}{ Cohort studies } \\
\hline \multirow[t]{2}{*}{$\begin{array}{l}\text { Cigarette } \\
\text { smoking }\end{array}$} & $\begin{array}{l}\text { Brooks } \\
\text { et al. [38], } \\
2004\end{array}$ & Face-to-face interview & Pregnancy & Maternal smoking & 144 & HR 1.24 (1.01-1.53) \\
\hline & $\begin{array}{l}\text { Tettamanti } \\
\text { et al. [37], } \\
2016\end{array}$ & Face-to-face interview & Pregnancy & Maternal smoking & $\begin{array}{l}147 \\
87\end{array}$ & $\begin{array}{l}\text { RR } 1.11(0.92-1.33) \text { for } 1-9 \text { cigs/day } \\
\text { RR } 1.15(0.91-1.44) \text { for } 10+\text { cigs/day }\end{array}$ \\
\hline \multirow[t]{2}{*}{$\begin{array}{l}\text { Parental } \\
\text { oc. }\end{array}$} & $\begin{array}{l}\text { Kristensen } \\
\text { et al. [67], } \\
1996\end{array}$ & $\begin{array}{l}\text { Information by } \\
\text { national agricultural } \\
\text { censuses }\end{array}$ & NA & Parental pesticide purchase & $\begin{array}{l}41 \\
22\end{array}$ & $\begin{array}{l}\text { RR } 1.71(1.11-2.63) \text { for BT aged } 0-14 \\
\text { RR } 3.37 \text { (1.63-6.94) for non-astrocytic } \\
\text { neuroepithelial tumours aged } 0-14\end{array}$ \\
\hline & $\begin{array}{l}\text { Spycher } \\
\text { et al. [68], } \\
2017\end{array}$ & Census records & Early childhood & $\begin{array}{l}\text { Maternal exposure to benzene } \\
\text { Paternal exposure to benzene }\end{array}$ & $\begin{array}{l}3 \\
4\end{array}$ & $\begin{array}{l}\text { HR } 0.22(0.03-1.60) \text { for gliomas } \\
\text { HR } 1.41(0.51-3.90) \text { for gliomas in the } \\
\text { highest exposure category }\end{array}$ \\
\hline \multicolumn{7}{|c|}{ Ecological study } \\
\hline \multirow[t]{3}{*}{$\begin{array}{l}\text { Air } \\
\text { pollution }\end{array}$} & \multirow[t]{3}{*}{$\begin{array}{l}\text { Danysh } \\
\text { et al. [8], } \\
2015\end{array}$} & \multirow[t]{3}{*}{$\begin{array}{l}\text { Concentrations by the } \\
\text { US environmental } \\
\text { protection agency } \\
\text { about participant's } \\
\text { residences }\end{array}$} & \multirow[t]{3}{*}{ During diagnosis } & 1,3-Butadiene & $\begin{array}{l}590 \\
117\end{array}$ & $\begin{array}{l}\text { IRR } 1.10(0.97-1.25) \text { for } \\
\left(0.031-0.046 \mu \mathrm{g} / \mathrm{m}^{3}\right) \text { concentration } \\
\text { IRR } 1.46(1.05-2.01) \text { for } \\
\left(0.031-0.046 \mu \mathrm{g} / \mathrm{m}^{3}\right) \text { concentration } \\
\text { in astrocytomas }\end{array}$ \\
\hline & & & & Benzene & 599 & $\begin{array}{l}\text { IRR } 1.07(0.94-1.21) \text { for } \\
\left(0.614-0.852 \mu \mathrm{g} / \mathrm{m}^{3}\right) \text { concentration }\end{array}$ \\
\hline & & & & DMP in residences & 657 & $\begin{array}{l}\text { IRR } 1.20(1.06-1.37) \text { for } \\
\left(0.218-0.784 \mu \mathrm{g} / \mathrm{m}^{3}\right) \text { concentration }\end{array}$ \\
\hline
\end{tabular}

* Means that the article has been also mentioned in another section. If tumour type is not specified it is all brain tumours.

Exp., exposure; Cd, cadmium; MBT, malignant brain tumours; CN, cranial nerves; BT, brain tumours; PNET, primitive neuroectodermal tumour; NA, not available; DMP, diesel particulate matter; HR, hazard ratio; IRR, incident rate ratio; PHA, polycyclic aromatic hydrocarbons.

analytical risk estimate for pesticides exposure during pregnancy was 1.73 (1.45-2.07), statistically significant, with no evidence of heterogeneity $\left(I^{2}=0 \%\right.$; online suppl. Fig. 3).

Results of exposure to pesticides during childhood or from prior to birth until BT diagnosis were mostly statistically not significant. Nevertheless, increases of risk were seen in $5[9,22,26,41,42]$ out of 8 studies which assessed exposure during childhood $[9,22,24-27,41$, 42]. These increases were seen in 2 studies of use of pesticides at home; 1 study of exposure to wood preservatives (53 cases and 746 controls) or home treated with pesticides once per year (8 cases and 96 controls) during childhood and risk of astrocytomas and 1 study of insecticides use by the father and risk of medulloblastomas or PNET (111 cases and 70 controls). A significantly increased meta-analytical effect estimate of 1.34 (1.151.56) was obtained for pesticide exposure during childhood. Heterogeneity was moderate $\left(I^{2}=60 \%\right.$; online suppl. Figure 3).

Another study that considered exposure starting 2 years before pregnancy until occurrence of BT in the offspring [43] found increased risks from use of herbicides at home and in combination with paternal occupational exposure for atrocytomas.

Eight studies analysed living on a farm and/or living with farm animals in different windows of exposure and BT risk. Five papers explored the association of childhood BT and mothers living and/or working on farms during pregnancy $[19,22,23,44,45]$. Increased risk was seen in 2 of them. A reduced risk was observed in 1 study $(n=352$ cases) if mothers lived on a farm during pregnancy [46]. A non-statistically significant increased meta-analytical risk estimate was obtained for studies with mothers living/working on a farm during pregnancy (1.17 [0.69$1.98])$. Heterogeneity was moderate $\left(I^{2}=63 \%\right.$; online suppl. Fig. 4).

Three articles explored mothers' contact with animals during pregnancy $[22,44,45]$ and found increased risks of BT in their offspring assessing different exposures: cat scratches, contact with pigs or living on a farm with animals.

Seven articles $[19,22,26,39,44-46]$ explored living on a farm during childhood as a risk factor for BT. Five of these articles suggested an increased risk, which was statistically significant in 3 (only one with large sample size) 
$[19,26,44]$. Two other articles found a non-statistically significant reduced risk, based on small sample sizes, for BT in relation to living or working on a farm during childhood. As for exposure to farm environment during pregnancy living on a farm during childhood resulted in a nonstatistical significant increased risk of $1.28(0.98-1.68)$ with moderate heterogeneity of $I^{2}=46 \%$ (online suppl. Fig. 4).

Four articles explored contact with farm animals and BT during childhood. Two articles found non-significantly increased risks: one for contact with sick pets (small sample) [26] and the other for exposure to any farm animal [44]. The other 2 articles found reduced risk in relation to contact with horses (small sample) [39], with birds (84 cases) or with goat and sheep (at least 3 years of exposure and 17 cases exposed) [46].

\section{Non-Ionizing Radiation}

Three articles [19, 42, 47] studied the association between extremely low frequency (ELF) exposure during pregnancy and BT risk.

Regarding the exposure to electrically heated waterbeds during pregnancy, 2 articles found non-significantly reduced risks ( 1 for astrocytomas and the other for BT in general) based on small samples sizes $[19,47]$. No significant association was found for PNET either [19]. Regarding exposure to ELF from electrical blankets during pregnancy, 3 articles found non-statistically significantly increased risk [19, 42, 47].

Two studies focused on childhood ELF exposure [39, 47] and BT risk. One [39] found a non-significantly reduced risk for electric blanket and waterbed use based on very small numbers of cases ( $n=6$ and 1 , respectively). In the other study, the ORs were close to one.

Two studies [48, 49] focused on radiofrequency (RF) exposures in childhood and BT were found. In the large multinational study [48], a non-significantly increased risk was found, with no evidence for an exposure response relationship between mobile phones use (which emits some ELF but mainly RF) and risk of BT. Use of mobile phones was low in this population, with an average duration of use of 2.7 years and lifetime cumulative time of use of only $35 \mathrm{~h}$. Another study [49], which estimated average of exposure to RF from mobile phone base stations, found a non-statistically significant risk for exposure above the median, though the authors acknowledge several methodological limitations.

Three studies focused on the exposure to magnetic fields (MF) [50-52] from high-current power lines, elec-

Environmental Factors and the Risk of BT

in Young People: A Systematic Review tric heating sources or electric appliances at home during childhood. One [50] found a significantly increased risk for $\geq 0.4 \mu \mathrm{T}$ of MF in the children's bedroom (OR 10.9 [1.05-113] based on 3 cases and 1 control). Two [51, 52] studies found a non-statistically significant increased risk for exposure at home to high MF compared to low ones.

\section{Parental Occupation}

Fourteen case - control and 2 cohort studies were found studying the association between parental occupational exposures and BT risk. Seven articles examined job exposures before child's birth [53-61]. All of them found increased risks of BT in the offspring for some occupations or occupational exposures reported by parents (factory worker, machine repair, painter, among others), although some non-statistically reduced risks were found for some paternal job exposures, including unspecified chemicals, hydrocarbons, electromagnetic fields, or metals among others and for mothers whose jobs were related to medicine and science in general, activities related to aerospace, agriculture and farming. Only 1 study with a small sample found an inverse association for astrocytomas and paternal job in transportation industry (OR $0.1[0.0-0.6])$ [57].

Seven articles explored a wider time window of exposures $[53,54,58-60,62,63]$. Three of them found associations with some parental exposures during the 5 years before child's birth. Another [62], with a small sample size, found a suggestion of an increased risk in children younger than 9 years old, and a non-significantly reduced risk in children between 10 and 15 years old, for paternal employment in the aerospace industry at least 1 year prior to birth until time of child's diagnosis. Another [63], found a statistically significant increased risk of astrocytoma in children whose mothers used insecticides in their jobs (53 cases and 35 controls).

Two other studies $[59,60]$ found a suggestion for an increased risk from mothers exposed to diesel or chlorinated solvents at any time before child's birth; statistically significant only in one of them (17 cases and 29 controls).

When considering exposure during the postnatal period, 4 articles [57, 64-66] found increased risk of BT, statistically significant in 3 (father exposed to electrical repairing, ionizing radiation, agriculture, etc.). Additionally, 2 of the studies found a suggestion for a reduced risk for astrocytomas with occupational exposures to metals or paints.

One of the 2 cohort studies that assessed parental occupation during childhood [67] found an increased risk for parental pesticide exposure. The other cohort study 
[68] found a non-significantly increased risk for paternal job exposure to benzene and a non-significantly reduced risk for maternal occupational exposure to benzene for astrocytomas. Exposures in this category were too heterogeneous to allow a formal meta-analysis.

\section{Diet}

Thirteen articles explored mother's diet during pregnancy and risk of BT in their offspring [22, 23, 25, 69-78]. Nine articles found an increased risk in relation to consumption of meat, which was statistically significant in 5 studies [23, 25, 69-71]. One of these studies [71] found an inverse association with BT and meat consumption during pregnancy if mothers also consumed multivitamin pills during all trimesters of pregnancy. Another article found a non-significantly reduced risk of BT related to meat consumption during pregnancy [22]. Other articles found reduced risk of BT in relation to consumption of iron supplements during pregnancy [72], consumption of vegetables $[69,73]$, grains or fresh fish [69] and non-fresh peaches or similar fruits [74]. The meta-analytical risk estimate for meat consumption during pregnancy was statistically significant and amounted to $1.51(1.32-1.73)$ though with some heterogeneity $\left(I^{2}=44 \%\right.$; online suppl. Fig. 5).

Increased risks of BT were found for consumption of French fries [74], bacon [73], non-cured meat or fresh fish [69] or hot-dogs [75].

Two articles found a non-significantly increased risk related to consumption of high levels of nitrate during childhood (calculated from reported food consumption) [76] or hot-dogs consumption one or more times per week [75]. The meta-analysis for these articles related to exposure during childhood was non-statistically significant with a meta-OR of $1.27(0.89-1.82)$ with no evidence of heterogeneity $\left(I^{2}=0 \%\right.$; online suppl. Fig. 5).

Another publication, not included in the tables, found an increased risk with increasing cured meat consumption during pregnancy in children without glutathione S-transferases theta 1 (OR 1.29 [1.07-1.57] for high cured meat intake per week) or reduced glutathione S-transferases mu 3 (OR 1.14 [1.03-1.26]). No increased risk was found for those with glutathione Stransferases theta 1 or normal glutathione S-transferases mu 3 levels [79].

Completeness of Methodological Information Reported When analysing the methodological information reporting completeness for the articles included in this review (online suppl. Table 3), we observed that a $97 \%$ of the articles included most of the 9 points that an article should include in the methodology section. The lowest score, due to missing information, was specifically concerning to participants' ethnicity, the recruitment period of the participants, number of controls matched per case, sample size and how missing data were managed. Sensitivity analyses were performed in few of the studies.

\section{Discussion/Conclusion}

Apart from ionising radiation and genetic syndromes, there are no established risk factors for BT in young people so far. Overall, there seems to exist some support in the literature for an association between BT and some specific environmental exposures. The early occurrence of BT suggests an important inherited component and that cancer initiating events might occur before conception, during foetal life or in early infancy [9], so the routes of exposure are diverse.

When considering exposures that can be associated with BT, it is particularly important to consider biological plausibility as the BBB protects the brain from toxicants. However, some toxicants have the capability of crossing the placenta and $\mathrm{BBB}$, and some of them, like some heavy metals, have known neurotoxic effects [80]. Furthermore, radiation (both ionising and non-ionising) can deposit energy in the brain.

Parents can be exposed at work and/or in their daily lives (through diet, water consumption, air pollution, etc.) to DNA-modifying agents that could affect the germ line. Also, some toxicants can accumulate in the body (e.g., some metals that have the ability to bioaccumulate in bones or pesticides in fatty tissue), and children may be exposed during mother's pregnancy [13] or after birth through breastfeeding. Also, during the first 5 years of life of the child, hand-to-mouth activities can be another source of exposure.

There are suggestions that exposure to certain metals can occur through: dental amalgams [81, 82], childhood vaccines that previously used the ethyl mercury compound thiomersal as a preservative [81], environmental tobacco smoke (exposing especially to $\mathrm{Cd}$ ), air pollution [81] or from young children eating paint chips in houses with old lead containing paints [83]. In our review, we found one article that suggested that cases with BT have higher concentrations of $\mathrm{Cd}$ in their biological samples than the control group [11], but no article assessing other metals, such as mercury, though 
children can be exposed to methyl mercury through diet (fish). A follow-up study found higher levels of $\mathrm{Cd}$ and lead ( $\mathrm{Pb}$; hair and toenails samples) in parents and BT cases compared to parents and children of the control group (low levels of metals) [84], and this was especially the case in children $<5$ years of age. Since we found only 1 article dealing with heavy metals and BT risk, there is insufficient evidence to support an association with BT.

Other toxicants related to cancer are disinfection byproducts, resulting from the treatment of drinking water from raw water containing organic matter. Trihalomethanes are the most studied disinfection by-products. We could not find any study about the relationship between BT risk and Trihalomethanes in young people, only few articles that suggest a possible association in adults [85]. Other water compound possibly related to cancer is nitrate, present in the water due to agricultural activity or wastewater treatment. Nitrate can be reduced to nitrite [86] compounds that are classified as probable human carcinogens. The articles found in this systematic review suggested an increased risk of BT in participants who consumed tap water with the presence of nitrite or nitrate. Nevertheless [10], an inverse association was found in Los Angeles region; the interpretation of this result is unclear, and is possibly due to low numbers of exposed: well water consumption was only reported by 20 participants of a total of 619 . The limit of detection of nitrite and nitrate levels is different between studies, preventing comparison and interpretation of the results.

Another source of exposure to nitrite and nitrate, besides drinking water, can be diet. Nitrate is a common preservative for meat. The majority of the articles about diet suggested an increased risk with higher meat consumption and a suggestion of reduced risk related to consumption of fruits [74], vegetables, grains and fish for astroglial tumours [69], or iron supplements and astrocytoma [72].

Tobacco smoke (classified as group 1 "carcinogenic to humans" by the International Agency for Research on Cancer) [87] contains many toxic compounds known to be related to cancer risk. Some articles suggested a relationship between smoking tobacco and alterations in the germ cells [88], childhood BT or with leukaemia-lymphoma [89]. Some tobacco smoke compounds can cross the BBB and placenta barrier [28]. These theoretical considerations can be supported by the results of 2 case-control and 1 cohort study finding significantly increased risk of BT in offspring whose mothers smoked, 7 studies for mother's ex-

Environmental Factors and the Risk of BT in Young People: A Systematic Review posure to passive smoking and one for exposure during the postnatal period. This associations evidence the possible genotoxic effects to the foetus and newborns.

Living in urban areas with high traffic density implies being exposed to different chemicals from vehicle exhausts. In this review, only 3 articles were found studying this association; specifically, 2 of them assessed exposure to 1,3-butadiene $[8,12]$, with increased risks, though focusing on exposure during different periods of child development.

Some pesticides have been banned due to their negative health implications. Nevertheless, some of them are very persistent and stay in the environment (especially soil and water) for many years. The use of pesticides continues to be quite common, specifically in agriculture, to sustain the current productivity. Other pesticides used by professional applicators are currently used for the treatment and maintenance of homes made of wood, or to exterminate common pests such as termites or rats. Some of the articles reviewed suggested an increased risk of childhood BT related to exposure during childhood as well as during the pregnancy period, especially for parental handling of pesticides and for professional exterminations at home or garden. Children can be exposed substantially since the home is the place where children generally spend most of their time.

We also evaluated exposures that can occur when living on a farm. Living on farms or with farm animals can imply exposure to a wide range of chemicals and other agents, including viruses and bacteria, that might explain the different results obtained in these studies. The articles suggested increased risk when mothers lived on a farm during pregnancy and also for exposures to some specific animals (such as pigs). An increased risk was also found related to children who lived on a farm during their early childhood.

The increasing use of new technologies and electrical devices has a high appeal among young people, and children are exposed at young ages. It is likely that, over their lifetime, children will accumulate more exposure to nonionising radiation than those who started using these technologies later in life. On the other hand, young people use, in general, more frequently messaging than calls to communicate, with a lower exposure to MF and RF received by the brain. In our systematic review, we found 6 articles that assessed exposure to non-ionizing radiation, and none of them found a significant association with BT, though most of them were based on small numbers and crude exposure estimates. 
Another source of exposure of the child or the foetus can be parental occupational exposures. Children are indirectly exposed during pregnancy from exposures of mothers in their jobs or during the postnatal period, through particles of paint, radionuclides or other products the parents may bring home on their clothes. We found some publications that suggested an increased risk related to some specific parental jobs. However, due to the fact that studies did not explore the same occupations or the same exposure period, a comparison is difficult and the evidence is not clear.

This is, to our knowledge, the first systematic review that includes all studies relating BT in young people to environmental risk factors up to June 2018. Since BT aetiology is largely unknown, it is important to have a better knowledge on possible risk factors.

This review has some limitations. The majority of the studies included were case - control studies with exposure assessment mostly based on personal interviews (with the possibility of differential recall bias between cases and controls) without measurements and, in most instances, no information provided about the analysis of possible exposure - response relationships. Some studies explored a great number of risk factors with an elevated risk of chance findings. In general, results are difficult to compare because of small sample sizes, different risk factors studied with different exposure indicators and different periods of exposure, and in some instances different outcomes (specific types of BTs). This might affect the strength of the evidence for establishing possible associations. A meta-analysis was therefore only possible for a limited number of exposures that were fairly consistent across studies. Another limitation is that some potentially eligible articles could have been missed due to shortcomings in search terms and phrases and as well the different strategies used for the databases.

Overall, the studies reviewed suggest that exposure to heavy metals, passive smoking, water and air pollutants, use of pesticides and living on a farm with farm animals, meat consumption, during preconception, pregnancy or early infancy may increase the risk of BT in children and young adults. However, inconsistencies of results, the small numbers of exposed cases in many studies, often crude exposure assessment and lack of detailed exposure response analyses, no clear conclusion can be drawn at present. Larger scale studies with better exposure assessment are needed to evaluate possible associations between environmental risk factors and BT in young people, especially on exposure to heavy metals, tap water consumption, pesticides and

parental smoking. Furthermore, exposure to RF electromagnetic fields and MF should receive more attention.

\section{Acknowledgements}

We would like to acknowledge all the studies included in this review. ISGlobal is a member of the CERCA Programme, Generalitat de Catalunya.

\section{Statements of Ethics}

The authors have no ethical conflicts to disclose.

\section{Disclosure Statement}

The authors have no conflicts of interest to declare.

\section{Financial Support}

The research leading to these results has received funding from the European Union Seventh Framework Programme FP7/20072013 under grant agreements n226873 (MOBI-Kids - Risk of brain cancer from exposure to RF fields in childhood and adolescencehttp://radiation.isglobal.org/mobi-kids) and 603794 (GERoNiMO - Generalized EMF Research Using Novel Methods http://radiation.isglobal.org/geronimo). The funding sources had no role in the planning and conduct of the study or in the interpretation or publication of the results.

\section{Author Contributions}

All authors of this research paper have directly participated in the planning and execution of the work, the analysis of the articles found, the interpretation of the findings and the writing of the manuscript. All authors of this paper have read and approved the final version submitted.

References

Neuroepidemiology 2019;53:121-141 DOI: $10.1159 / 000500601$
Zumel-Marne/Castano-Vinyals/Kundi/ Alguacil/Cardis
1 IARC. IARC Press Release 241. International Childhood Cancer Day: Much remains to be done to fight childhood [cited 2017 Mar 10]. Available from: https://www.iarc.fr/en/media-centre/pr/2016/pdfs/pr241_E.pdf.

2 Subramanian S, Ahmad T. Cancer, Childhood Brain Tumors. In: StatPearls [cited 2019 Apr 11]. Treasure Island, FL: StatPearls Publishing; 2019. Available from: http://www. ncbi.nlm.nih.gov/books/NBK535415/.

3 Ward E, DeSantis C, Robbins A, Kohler B, Jemal A. Childhood and adolescent cancer statistics, 2014. CA Cancer J Clin. 2014 Mar-Apr; 64(2):83-103. 
4 Stewart BW, Wild CP, editors. World Cancer Report 2014. Lyon, France: International Agency for Research on Cancer; 2014.

5 Hemminki K, Li X. Cancer risks in childhood and adolescence among the offspring of immigrants to Sweden. Br J Cancer. 2002 May; 86(9):1414-8

6 Black PM, Loeffler JS. Cancer of the Nervous System. Lippincott Williams \& Wilkins; 2005.

7 Cordier S, Monfort C, Filippini G, PrestonMartin S, Lubin F, Mueller BA, et al. Parental exposure to polycyclic aromatic hydrocarbons and the risk of childhood brain tumors: The SEARCH International Childhood Brain Tumor Study. Am J Epidemiol. 2004 Jun; 159(12):1109-16

8 Danysh HE, Mitchell LE, Zhang K, Scheurer ME, Lupo PJ. Traffic-related air pollution and the incidence of childhood central nervous system tumors: Texas, 2001-2009. Pediatr Blood Cancer. 2015 Sep;62(9):1572-8.

9 Greenop KR, Peters S, Bailey HD, Fritschi L, Attia J, Scott RJ, et al. Exposure to pesticides and the risk of childhood brain tumors. Cancer Causes Control. 2013 Jul;24(7):1269-78.

10 Mueller BA, Searles Nielsen S, Preston-Martin S, Holly EA, Cordier S, Filippini G, et al. Household water source and the risk of childhood brain tumours: results of the SEARCH International Brain Tumor Study. Int J Epidemiol. 2004 Dec;33(6):1209-16.

11 Sherief LM, Abdelkhalek ER, Gharieb AF, Sherbiny HS, Usef DM, Almalky MA, et al. Cadmium status among pediatric cancer patients in Egypt. Medicine (Baltimore). 2015 May;94(20):e740.

12 Von Ehrenstein OS, Heck JE, Park A, Cockburn M, Escobedo L, Ritz B. In utero and Early-Life Exposure to Ambient Air Toxics and Childhood Brain Tumors: A PopulationBased Case-Control Study in California, USA. Environ Health Perspect. 2016 Jul;124(7): 1093-9.

13 Martins E, Varea A, Apezteguía M, González HF, Girardelli A, Caro LS, et al. Prenatal lead exposure and relationship with maternal exposure determinants in a public maternity hospital of La Plata, Argentina. Sci Total Environ. 2014 Mar;473-474:43-7.

14 Vandenbroucke JP, von Elm E, Altman DG, Gøtzsche PC, Mulrow CD, Pocock SJ, et al.; STROBE initiative. Strengthening the Reporting of Observational Studies in Epidemiology (STROBE): explanation and elaboration. Ann Intern Med. 2007 Oct;147(8):W163-94.

15 Mueller BA, Newton K, Holly EA, PrestonMartin S. Residential water source and the risk of childhood brain tumors. Environ Health Perspect. 2001 Jun;109(6):551-6.

16 Weng HH, Tsai SS, Wu TN, Sung FC, Yang $\mathrm{CY}$. Nitrates in drinking water and the risk of death from childhood brain tumors in Taiwan. J Toxicol Environ Health A. 2011; 74(12):769-78

17 Filippini G, Farinotti M, Ferrarini M. Active and passive smoking during pregnancy and risk of central nervous system tumours in children. Paediatr Perinat Epidemiol. 2000 Jan;14(1):78-84.

18 Filippini G, Farinotti M, Lovicu G, Maisonneuve $\mathrm{P}$, Boyle P. Mothers' active and passive smoking during pregnancy and risk of brain tumours in children. Int J Cancer. 1994 Jun; 57(6):769-74.

19 Bunin GR, Buckley JD, Boesel CP, Rorke LB, Meadows AT. Risk factors for astrocytic glioma and primitive neuroectodermal tumor of the brain in young children: a report from the Children's Cancer Group. Cancer Epidemiol Biomarkers Prev. 1994 Apr-May;3(3):197-204.

20 Milne E, Greenop KR, Scott RJ, Ashton LJ, Cohn RJ, de Klerk NH, et al. Parental smoking and risk of childhood brain tumors. Int J Cancer. 2013 Jul;133(1):253-9.

21 Filippini G, Maisonneuve P, McCredie M, Peris-Bonet R, Modan B, Preston-Martin S, et al. Relation of childhood brain tumors to exposure of parents and children to tobacco smoke: the SEARCH international case-control study. Surveillance of Environmental Aspects Related to Cancer in Humans. Int J Cancer. 2002 Jul;100(2):206-13.

22 Cordier S, Iglesias MJ, Le Goaster C, Guyot MM, Mandereau L, Hemon D. Incidence and risk factors for childhood brain tumors in the Ile de France. Int J Cancer. 1994 Dec;59(6): 776-82.

23 McCredie M, Maisonneuve P, Boyle P. Antenatal risk factors for malignant brain tumours in New South Wales children. Int J Cancer. 1994 Jan;56(1):6-10.

24 Pogoda JM, Preston-Martin S. Household pesticides and risk of pediatric brain tumors. Environ Health Perspect. 1997 Nov; 105(11): 1214-20.

25 Preston-Martin S, Yu MC, Benton B, Henderson BE. N-Nitroso compounds and childhood brain tumors: a case-control study. Cancer Res. 1982 Dec;42(12):5240-5.

26 Gold E, Gordis L, Tonascia J, Szklo M. Risk factors for brain tumors in children. Am J Epidemiol. 1979 Mar;109(3):309-19.

27 Howe GR, Burch JD, Chiarelli AM, Risch HA, Choi BC. An exploratory case-control study of brain tumors in children. Cancer Res. 1989 Aug;49(15):4349-52.

28 Norman MA, Holly EA, Preston-Martin S. Childhood brain tumors and exposure to tobacco smoke. Cancer Epidemiol Biomarkers Prev. 1996 Feb;5(2):85-91.

29 John EM, Savitz DA, Sandler DP. Prenatal exposure to parents' smoking and childhood cancer. Am J Epidemiol. 1991 Jan;133(2):123-32.

30 Gold EB, Leviton A, Lopez R, Gilles FH, Hedley-Whyte ET, Kolonel LN, et al. Parental smoking and risk of childhood brain tumors. Am J Epidemiol. 1993 Mar;137(6):620-8.

31 Ji BT, Shu XO, Linet MS, Zheng W, Wacholder S, Gao YT, et al. Paternal cigarette smoking and the risk of childhood cancer among offspring of nonsmoking mothers. J Natl Cancer Inst. $1997 \mathrm{Feb} ; 89(3): 238-44$.

$32 \mathrm{Hu}$ J, Mao Y, Ugnat AM; Jinfu Hu, Yang Mao, Anne-Marie Ugna. Parental cigarette smok- ing, hard liquor consumption and the risk of childhood brain tumors-a case-control study in northeast China. Acta Oncol. 2000;39(8): 979-84.

33 Pavlovic MV, Jarebinski MS, Pekmezovic TD, Janicijevic MA. Risk factors from brain tumors in children and adolescents: a case-control study in Belgrade, Serbia. Eur J Neurol. 2005 Jul;12(7):508-13.

34 Plichart M, Menegaux F, Lacour B, Hartmann O, Frappaz D, Doz F, et al. Parental smoking, maternal alcohol, coffee and tea consumption during pregnancy and childhood malignant central nervous system tumours: the ESCALE study (SFCE). Eur J Cancer Prev. 2008 Aug; 17(4):376-83.

35 Barrington-Trimis JL, Searles Nielsen S, Preston-Martin S, Gauderman WJ, Holly EA, Farin $\mathrm{FM}$, et al. Parental smoking and risk of childhood brain tumors by functional polymorphisms in polycyclic aromatic hydrocarbon metabolism genes. PLoS One. 2013 Nov; 8(11):e79110.

36 Vienneau D, Infanger D, Feychting M, Schüz J, Schmidt LS, Poulsen AH, et al. A multinational case-control study on childhood brain tumours, anthropogenic factors, birth characteristics and prenatal exposures: A validation of interview data. Cancer Epidemiol. 2016 Feb;40:52-9.

37 Tettamanti G, Ljung R, Mathiesen $T$, Schwartzbaum J, Feychting M. Maternal smoking during pregnancy and the risk of childhood brain tumors: results from a Swedish cohort study. Cancer Epidemiol. 2016 Feb; 40:67-72.

38 Brooks DR, Mucci LA, Hatch EE, Cnattingius S. Maternal smoking during pregnancy and risk of brain tumors in the offspring. A prospective study of 1.4 million Swedish births. Cancer Causes Control. 2004 Dec;15(10): 997-1005.

39 McCredie M, Maisonneuve P, Boyle P. Perinatal and early postnatal risk factors for malignant brain tumours in New South Wales children. Int J Cancer. 1994 Jan;56(1):11-5.

40 Danysh HE, Zhang K, Mitchell LE, Scheurer ME, Lupo PJ. Maternal residential proximity to major roadways at delivery and childhood central nervous system tumors. Environ Res. 2016 Apr;146:315-22.

41 Rosso AL, Hovinga ME, Rorke-Adams LB, Spector LG, Bunin GR; Children's Oncology Group. A case-control study of childhood brain tumors and fathers' hobbies: a Children's Oncology Group study. Cancer Causes Control. 2008 Dec;19(10):1201-7.

42 Schüz J, Kaletsch U, Kaatsch P, Meinert R, Michaelis J. Risk factors for pediatric tumors of the central nervous system: results from a German population-based case-control study. Med Pediatr Oncol. 2001 Feb;36(2):274-82.

43 Shim YK, Mlynarek SP, van Wijngaarden E. Parental exposure to pesticides and childhood brain cancer: U.S. Atlantic coast childhood brain cancer study. Environ Health Perspect. 2009 Jun;117(6):1002-6.
Environmental Factors and the Risk of BT in Young People: A Systematic Review
Neuroepidemiology 2019;53:121-141 DOI: $10.1159 / 000500601$ 
44 Efird JT, Holly EA, Preston-Martin S, Mueller BA, Lubin F, Filippini G, et al. Farm-related exposures and childhood brain tumours in seven countries: results from the SEARCH International Brain Tumour Study. Paediatr Perinat Epidemiol. 2003 Apr;17(2):201-11.

45 Holly EA, Bracci PM, Mueller BA, PrestonMartin S. Farm and animal exposures and pediatric brain tumors: results from the United States West Coast Childhood Brain Tumor Study. Cancer Epidemiol Biomarkers Prev. 1998 Sep;7(9):797-802.

46 Christensen JS, Mortensen LH, Röösli M, Feychting M, Tynes T, Andersen TV, et al. Brain tumors in children and adolescents and exposure to animals and farm life: a multicenter case-control study (CEFALO). Cancer Causes Control. 2012 Sep;23(9):1463-73.

47 Preston-Martin S, Gurney JG, Pogoda JM, Holly EA, Mueller BA. Brain tumor risk in children in relation to use of electric blankets and water bed heaters. Results from the United States West Coast Childhood Brain Tumor Study. Am J Epidemiol. 1996 Jun;143(11): 1116-22.

48 Aydin D, Feychting M, Schüz J, Tynes T, Andersen TV, Schmidt LS, et al. Mobile phone use and brain tumors in children and adolescents: a multicenter case-control study. J Natl Cancer Inst. 2011 Aug;103(16):1264-76.

49 Li CY, Liu CC, Chang YH, Chou LP, Ko MC. A population-based case-control study of radiofrequency exposure in relation to childhood neoplasm. Sci Total Environ. 2012 Oct; 435-436:472-8.

50 Saito T, Nitta H, Kubo O, Yamamoto S, Yamaguchi N, Akiba S, et al. Power-frequency magnetic fields and childhood brain tumors: a case-control study in Japan. J Epidemiol. 2010;20(1):54-61.

51 Gurney JG, Mueller BA, Davis S, Schwartz SM, Stevens RG, Kopecky KJ. Childhood brain tumor occurrence in relation to residential power line configurations, electric heating sources, and electric appliance use. Am J Epidemiol. 1996 Jan;143(2):120-8.

52 Savitz DA, Wachtel H, Barnes FA, John EM, Tvrdik JG. Case-control study of childhood cancer and exposure to $60-\mathrm{Hz}$ magnetic fields. Am J Epidemiol. 1988 Jul;128(1):21-38.

53 Cordier S, Mandereau L, Preston-Martin S, Little J, Lubin F, Mueller B, et al. Parental occupations and childhood brain tumors: results of an international case-control study. Cancer Causes Control. 2001 Nov;12(9):865-74.

54 Cordier S, Lefeuvre B, Filippini G, Peris-Bonet R, Farinotti M, Lovicu G, et al. Parental occupation, occupational exposure to solvents and polycyclic aromatic hydrocarbons and risk of childhood brain tumors (Italy, France, Spain). Cancer Causes Control. 1997 Sep;8(5):688-97.

55 Feingold L, Savitz DA, John EM. Use of a jobexposure matrix to evaluate parental occupation and childhood cancer. Cancer Causes Control. 1992 Mar;3(2):161-9.

56 Hemminki K, Saloniemi I, Salonen T, Partanen T, Vainio H. Childhood cancer and pa- rental occupation in Finland. J Epidemiol Community Health. 1981 Mar;35(1):11-5.

57 Kuijten RR, Bunin GR, Nass CC, Meadows AT. Parental occupation and childhood astrocytoma: results of a case-control study. Cancer Res. 1992 Feb;52(4):782-6.

58 McKean-Cowdin R, Preston-Martin S, Pogoda JM, Holly EA, Mueller BA, Davis RL. Parental occupation and childhood brain tumors: astroglial and primitive neuroectodermal tumors. J Occup Environ Med. 1998 Apr;40(4):332-40.

59 Peters S, Glass DC, Greenop KR, Armstrong BK, Kirby M, Milne E, et al. Childhood brain tumours: associations with parental occupational exposure to solvents. Br J Cancer. 2014 Aug;111(5):998-1003.

60 Peters S, Glass DC, Reid A, de Klerk N, Armstrong BK, Kellie S, et al. Parental occupational exposure to engine exhausts and childhood brain tumors. Int J Cancer. 2013 Jun;132(12): 2975-9.

61 Wilkins JR 3rd, Sinks T. Parental occupation and intracranial neoplasms of childhood: results of a case-control interview study. Am J Epidemiol. 1990 Aug;132(2):275-92.

62 Olshan AF, Breslow NE, Daling JR, Weiss NS, Leviton A. Childhood brain tumors and paternal occupation in the aerospace industry. J Natl Cancer Inst. 1986 Jul;77(1):17-9.

63 van Wijngaarden E, Stewart PA, Olshan AF, Savitz DA, Bunin GR. Parental occupational exposure to pesticides and childhood brain cancer. Am J Epidemiol. 2003 Jun;157(11): 989-97.

64 Keegan TJ, Bunch KJ, Vincent TJ, King JC, O’Neill KA, Kendall GM, et al. Case-control study of paternal occupation and social class with risk of childhood central nervous system tumours in Great Britain, 1962-2006. Br J Cancer. 2013 May;108(9):1907-14.

65 Nasca PC, Baptiste MS, MacCubbin PA, Metzger BB, Carlton K, Greenwald P, et al. An epidemiologic case-control study of central nervous system tumors in children and parental occupational exposures. Am J Epidemiol. 1988 Dec;128(6):1256-65.

66 Wilkins JR 3rd, Koutras RA. Paternal occupation and brain cancer in offspring: a mortality-based case-control study. Am J Ind Med. 1988;14(3):299-318

67 Kristensen P, Andersen A, Irgens LM, Bye AS, Sundheim L. Cancer in offspring of parents engaged in agricultural activities in Norway: incidence and risk factors in the farm environment. Int J Cancer. 1996 Jan;65(1): 39-50.

68 Spycher BD, Lupatsch JE, Huss A, Rischewski J, Schindera C, Spoerri A, et al.; Swiss Paediatric Oncology Group; Swiss National Cohort Study Group. Parental occupational exposure to benzene and the risk of childhood cancer: A census-based cohort study. Environ Int. 2017 Nov; 108:84-91.

69 Pogoda JM, Preston-Martin S, Howe G, Lubin F, Mueller BA, Holly EA, et al. An international case-control study of maternal diet during pregnancy and childhood brain tumor risk: a histology-specific analysis by food group. Ann Epidemiol. 2009 Mar;19(3):148-60.

70 Pogoda JM, Preston-Martin S. Maternal cured meat consumption during pregnancy and risk of paediatric brain tumour in offspring: potentially harmful levels of intake. Public Health Nutr. 2001 Apr;4(2):183-9.

71 Preston-Martin S, Pogoda JM, Mueller BA, Holly EA, Lijinsky W, Davis RL. Maternal consumption of cured meats and vitamins in relation to pediatric brain tumors. Cancer Epidemiol Biomarkers Prev. 1996b Aug;5(8): 599-605.

72 Bunin GR, Kuijten RR, Boesel CP, Buckley JD, Meadows AT. Maternal diet and risk of astrocytic glioma in children: a report from the Childrens Cancer Group (United States and Canada). Cancer Causes Control. 1994b Mar;5(2):177-87.

73 Bunin GR, Kuijten RR, Buckley JD, Rorke LB, Meadows AT. Relation between maternal diet and subsequent primitive neuroectodermal brain tumors in young children. $\mathrm{N}$ Engl $\mathrm{J}$ Med. 1993 Aug;329(8):536-41.

74 Bunin GR, Kushi LH, Gallagher PR, RorkeAdams LB, McBride ML, Cnaan A. Maternal diet during pregnancy and its association with medulloblastoma in children: a children's oncology group study (United States). Cancer Causes Control. 2005 Sep;16(7):877-91.

75 Sarasua S, Savitz DA. Cured and broiled meat consumption in relation to childhood cancer: Denver, Colorado (United States). Cancer Causes Control. 1994 Mar;5(2):141-8.

76 Lubin F, Farbstein H, Chetrit A, Farbstein M, Freedman L, Alfandary E, et al. The role of nutritional habits during gestation and child life in pediatric brain tumor etiology. Int J Cancer. 2000 Apr;86(1):139-43.

77 Bunin GR, Gallagher PR, Rorke-Adams LB, Robison LL, Cnaan A. Maternal supplement, micronutrient, and cured meat intake during pregnancy and risk of medulloblastoma during childhood: a children's oncology group study. Cancer Epidemiol Biomarkers Prev. 2006 Sep;15(9):1660-7.

78 Greenop KR, Miller M, Attia J, Ashton LJ, Cohn R, Armstrong BK, et al. Maternal consumption of coffee and tea during pregnancy and risk of childhood brain tumors: results from an Australian case-control study. Cancer Causes Control. 2014 Oct;25(10):13217.

79 Searles Nielsen S, Mueller BA, Preston-Martin S, Farin FM, Holly EA, McKean-Cowdin R. Childhood brain tumors and maternal cured meat consumption in pregnancy: differential effect by glutathione S-transferases. Cancer Epidemiol Biomarkers Prev. 2011 Nov;20(11):2413-9.

80 Goyer RA. Transplacental transport of lead. Environ Health Perspect. 1990 Nov;89:101-5.

81 Borchers A, Teuber SS, Keen CL, Gershwin ME. Food safety. Clin Rev Allergy Immunol. 2010 Oct;39(2):95-141.

82 Järup L. Hazards of heavy metal contamination. Br Med Bull. 2003;68(1):167-82. 
83 Carpenter DO. Effects of metals on the nervous system of humans and animals. Int J Occup Med Environ Health. 2001;14(3): 209-18.

84 Wilhelm M, Lombeck I, Ohnesorge FK. Cadmium, copper, lead and zinc concentrations in hair and toenails of young children and family members: a follow-up study. Sci Total Environ. 1994 Jan;141(1-3): 275-80.

85 Cantor KP, Lynch CF, Hildesheim ME, Dosemeci M, Lubin J, Alavanja M, et al. Drinking water source and chlorination byproducts in Iowa. III. Risk of brain cancer. Am J Epidemiol. 1999 Sep;150(6):55260.

86 Wold Health Organization (WHO). Beryllium in drinking-water: background document for development of WHO guidelines for drinking-water quality 2009 [cited $2016 \mathrm{Apr}$ 25]. Available from: http://apps.who.int/iris/ handle/10665/70172.

87 IARC. Agents classified by the IARC monographs, volumes 1-121. Lyon, France: International Agency for Research on Cancer 2018 [cited 2016 Apr 24]. Available from: http://
monographs.iarc.fr/ENG/Classification/index.php.

88 Esakky P, Hansen DA, Drury AM, Felder P, Cusumano A, Moley KH. Paternal exposure to cigarette smoke condensate leads to reproductive sequelae and developmental abnormalities in the offspring of mice. Reprod Toxicol. 2016 Oct; 65:283-94.

89 Sasco AJ, Vainio H. From in utero and childhood exposure to parental smoking to childhood cancer: a possible link and the need for action. Hum Exp Toxicol. 1999 Apr;18(4): 192-201. 